Accepted refereed manuscript of:

Paton MD, Green SF, Ball AJ, Zarnecki JC \& Hagermann A (2016) Detection of structure in asteroid analogue materials and Titan's regolith by a landing spacecraft, Advances in Space Research, 58 (3), pp. 415-437.

DOI: 10.1016/j.asr.2016.04.026

(C) 2016, Elsevier. Licensed under the Creative Commons AttributionNonCommercial-NoDerivatives 4.0 International http://creativecommons.org/licenses/by-nc-nd/4.0/ 


\section{Accepted Manuscript}

Detection of structure in asteroid analogue materials and Titan's regolith by a

landing spacecraft

M.D. Paton, S.F. Green, A.J. Ball, J.C. Zarnecki, A. Hagermann

PII:

S0273-1177(16)30161-2

DOI:

http://dx.doi.org/10.1016/j.asr.2016.04.026

Reference:

JASR 12711

To appear in: $\quad$ Advances in Space Research

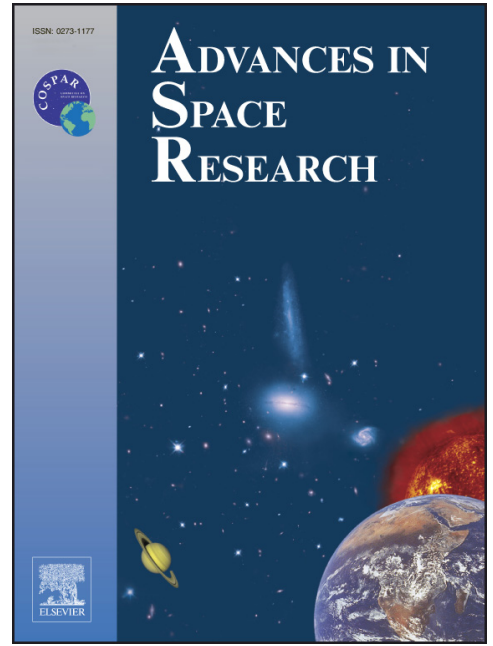

Received Date: $\quad 5$ October 2015

Revised Date: $\quad 21$ March 2016

Accepted Date: $\quad 26$ April 2016

Please cite this article as: Paton, M.D., Green, S.F., Ball, A.J., Zarnecki, J.C., Hagermann, A., Detection of structure in asteroid analogue materials and Titan's regolith by a landing spacecraft, Advances in Space Research (2016), doi: http://dx.doi.org/10.1016/j.asr.2016.04.026

This is a PDF file of an unedited manuscript that has been accepted for publication. As a service to our customers we are providing this early version of the manuscript. The manuscript will undergo copyediting, typesetting, and review of the resulting proof before it is published in its final form. Please note that during the production process errors may be discovered which could affect the content, and all legal disclaimers that apply to the journal pertain. 


\title{
Detection of structure in asteroid analogue materials and Titan's regolith by a landing spacecraft
}

\author{
M. D. Paton** \\ Finnish Meteorological Institute, PO Box 503, FIN-00101 Helsinki, Finland \\ S. F. Green, A. J. Ball \\ Department of Physical Sciences, The Open University, Milton Keynes, MK7 6AA, UK \\ J. C. Zarnecki \\ International Space Science Institute, 3012 Bern, Switzerland \\ A. Hagermann \\ Department of Physical Sciences, The Open University, Milton Keynes, MK7 6AA, UK
}

\section{Abstract}

We compare measurements made by two impact penetrometers of different sizes and with different tip shapes to further understand penetrometer design for performing pentrometry on an asteroid. To this end we re-visit the interpretation of data from the Huygens' penetrometer, ACC-E, that impacted Titan's surface. In addition we investigate the potential of a spacecraft fitted with a penetrometer to bounce using a test rig, built at The Open University (UK).

Analysis of ACC-E laboratory data, obtained from impacts into $\sim 4 \mathrm{~mm}$ diameter gravel, was found to produce an unusual decrease in resistance with depth (force-depth gradient) which was also seen in the Huygens' ACC$\mathrm{E}$ data from Titan and originally interpreted as a wet or moist sand. The downward trend could also be reproduced in a hybrid Discrete Element Model (DEM) if it was assumed that the near surface particles are more readily

${ }^{*}$ Corresponding author

** Phone: +358 504302984

Email address: mark.paton@fmi.fi (M. D. Paton ) 
mobilised than those deeper in the target.

With regard to penetrometer design penetration resistance was found to be sensitive to the ratio of particle to tip diameter. A clear trend was observed with a conical tip penetrometer, X-PEN, of decreasing force-depth gradients with increasing particle sizes most likely due to a transformation from a bulk displacement of material by the penetrating tip to more local interactions. ACC-E, which has a hemispherical tip, was found to produce a wider range of force-depth gradients than X-PEN, which had a conical tip, possibly due to difficulties dislodging jammed particles. Both penetrometers were able to determine particle diameter and mass after post-processing of the data.

Laboratory simulations of landings with the test rig suggest that a large impact penetrometer under certain circumstances could absorb a significant amount of the elastic energy of the spacecraft possibly aiding landing. Alternatively a small impact penetrometer would allow the spacecraft to bounce freely off the surface to make a measurement at another location.

Keywords: spacecraft, impact, Huygens, regolith, penetrometer, asteroid

\section{Introduction}

In situ investigation of the surface layer and subsurface strata of a planetary body can reveal a record of geological activity (Moore \& Jakosky, 1989; Zarnecki et al., 2005; Yano et al., 2006; Keller et al., 2008) while the microstructural properties of the particles provide an insight into the interpretation of these layers and the relative importance of the processes involved in their formation (Burr et al., 2006; Sullivan et al., 2008). A variety of techniques have been used for in situ exploration of the subsurface such as static penetrometers, impact penetrometers, penetrators and drills. The dynamics of landing spacecraft is often used as a way to determine the mechanical properties of the surface (Kömle et al., 2001; Kargl et al., 2009; Ball et al., 2010).

A conventional spacecraft with a mass of around $100 \mathrm{~kg}$ landing at a few metres per second, with a penetrometer fitted to its base, has enough momentum to deliver sensors into the sub-surface of an asteroid or other planetary regolith by virtue of its high mass (Lorenz et al., 1994; Paton et al., 2015). Despite the low speed, the high mass of the spacecraft presents a momentum comparable to, if not exceeding, the values obtained by low 


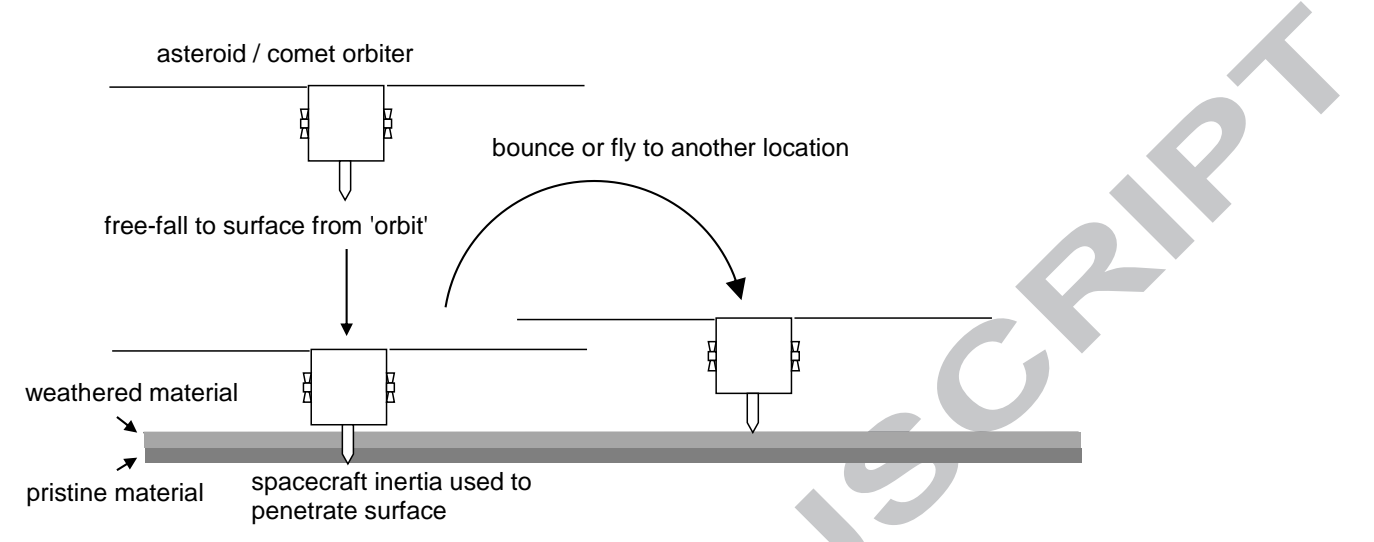

Figure 1: Impact penetrometry by a bouncing spacecraft.

mass, high speed penetrators. An impact penetrometer is normally limited to performing a single measurement, i.e. on landing.

On a low gravity body such as an asteroid or comet the measurement could be performed a few times as it bounces across the surface or more if it has a propulsion system, e.g. see figure 1. Such measurements could be useful for investigating slope processes and other regolith possible as was possible with the Lunokhod lunar rover (Cherkasov \& Shvarev). Lunokhod's penetrometer was actuated at several different point and relied on the weight of the rover provide a stable platform. On an asteroid such an actuated penetrometer deployment may not work due to the low gravitational acceleration and the low weight of the rover. Impact penetrometry solves this issue by using the momentum of the spacecraft to deploy the penetrometer rather than relying on the presence of a stable platform to push against.

If a rebound is not desired some active landing approach, e.g. Chitu et al. (2015), would be required to maintain contact with the surface. It may also be possible to use a large rigid penetrometer to aid the landing as explored by Paton et al. (2015) to absorb some of the elastic energy of the spacecraft during landing under certain circumstances.

The Huygens spacecraft performed an impact penetrometry measurement on the surface of Titan as part of the Surface Science Package (Zarnecki et al., 2002). The penetrometry data was found to be consistent with a moderately firm granular material, possibly wet. The measurement was considered repre- 
sentative of the subsurface down to a depth of $5 \mathrm{~cm}$ after which the impact of the spacecraft main body modified the penetration mechanics in an unknown manner (Zarnecki et al., 2005; Atkinson et al., 2010). A notable feature in the penetrometry data, over the first five centimetres of penetration, is a negative force-depth gradient that Atkinson et al. (2010) reproduced in the laboratory with penetrometer impacts into coarse wet sand. Atkinson et al. (2010) also investigated the particle size at the Huygens site and found that their data was consistent with a particle diameter of $2 \mathrm{~mm}$ but noted that larger particles may exist at the site as seen in the Huygens imager data (Keller et al., 2008).

In this paper we explore some aspects of penetrometer design, fitted to the base of spacecraft, to detect structures in the regolith of planetary bodies. To this end we re-examine the Huygens penetrometer data by performing laboratory tests of a penetrometer in coarse-grained materials and offer an alternative to the wet sand interpretation. We also investigate the potential of a landing spacecraft fitted with an impact penetrometer to bounce of the surface. In section 2 some background on impact penetrometry and the penetrometer signal from Huygens is provided. In section 3 the test rig apparatus, instrumentation and operation is described. In section 4 we outline the numerical models used to interpret the diagnostic instruments on the test rig and interpret the penetrometry measurements. In section 5 the results of impact penetrometry of layered materials and of coarse-grained materials are presented. The penetrometry signal from Huygens is compared to laboratory tests and a hybrid DEM.

\section{Regolith structure and microstructure measured by a landing spacecraft}

Geophysical investigations of terrestrial soils (Paillet \& Saunders, 1990) use a variety of in situ and laboratory measurements to explore the subsurface structure and its response to disturbances. In situ tests down to a depth of several metres may include digging trenches and test pits and performing penetration tests. Bore holes may be drilled and sensors place in them. For depths down to several tens of metres the application of seismic refraction methods is required. Soil collected in the field can be tested in the laboratory under controlled conditions using a number of standardised procedures that may include shear tests and the Split-Hopkinson force bar test. Typical 
properties that these tests can determine are particle properties cohesion, internal friction and the response of a soil to impulse loads.

A processed top layer will often be present on a planetary surface, masking the more representative material below from remote observation techniques, e.g. Putzig et al. (2005). A way to reach the relatively pristine shallow subsurface is to use a penetrometer (Kömle et al., 2001; Kargl et al., 2009) or drill (Spohn et al., 2007; Poletto et al., 2015; Seweryn et al., 2014). These can determine the mechanical properties of the regolith to potentially investigate the subsurface strata and infer microstructural properties of the particles. Penetrometers and drills also have the potential to deliver instruments into the subsurface to further investigate the subsurface. The signal from a penetrometer can be processed to obtain regolith properties such as internal friction, cohesion and particle size, e.g. Paton et al. (2012a, 2015).

A simple and robust method of deploying a penetrometer into the subsurface is to fix it to the base of a landing spacecraft. The low speed but high mass of the lander will then push the penetrometer into the shallow subsurface. This technique was used by the Huygens landing on Titan (Lorenz et al., 1994; Zarnecki et al., 2005). Impact penetrometry is particularly valuable when visiting a body for the first time (Jones, 1971; Spohn et al., 2015). A limitation of the technique is that it performs a 'single point' measurement that may not be representative of the region around the lander. However, information from additional instruments such as sonar and imaging can help put the measurement into context, e.g. Zarnecki et al. (2005).

Figure 2 shows the penetrometry signal returned by the Huygens penetrometer. The prominent features of the data, relevant to this study, are labelled. The feature labelled $\mathrm{A}$ is a low strength material that has been associated with a loose covering of deposited material (Atkinson et al., 2010) most likely airfall dust (Schröder et al., 2012). The feature labelled B is a peak, possibly caused by the impact with one of the ice pebbles observed on the surface. The feature labelled $\mathrm{C}$ is a negative force-depth profile, which is the main focus of this study. It has been previously investigated by Atkinson et al. (2010) who found that wet coarse sand had similar mechanical properties to the surface of Titan. The feature labelled D is the point where the main body impacts the surface and changes the surface's mechanical response to the penetrometer in an unknown way. It is tempting to label the features as discrete stages but we would like to maintain the possibility that the features are due to penetrometer-material interactions rather than purely the result of the regolith properties. 


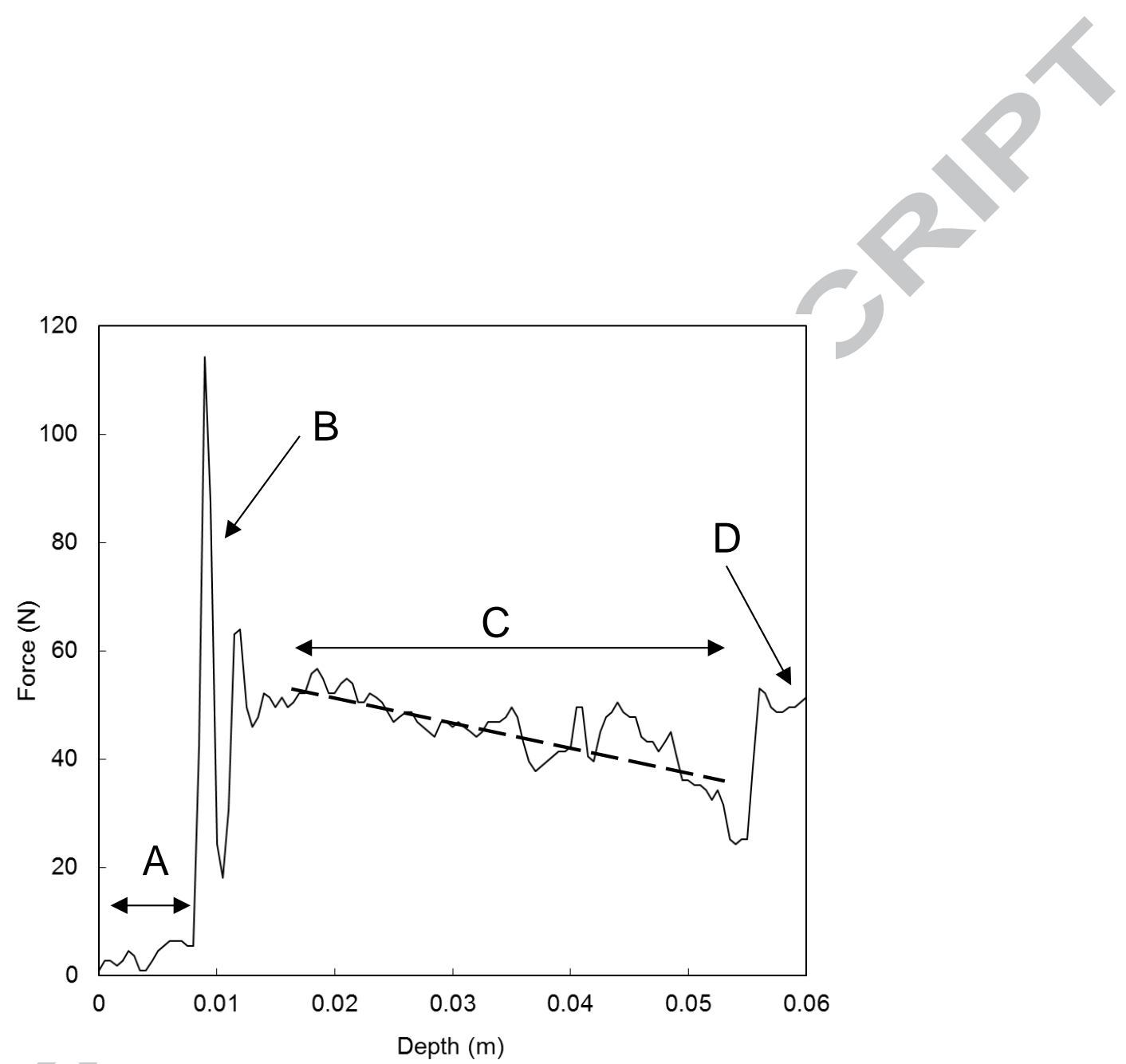

Figure 2: Penetrometer signal returned by Huygens from Titan. Pertinent features in the data related to this study are labelled. 


\section{The test rig}

Landings of spacecraft in a low gravity environment are often simulated in the laboratory for shock testing and landing gear modelling impacting a solid surface. Commonly used methods are scale models, pendulums with inclined planes, sideways impacts and using weights to balance out Earth's gravity (Seiff et al., 2005; Hilchenbach et al., 2004; Reid et al., 2014). Impacts of projectiles and small spacecraft (cm sized) into analogue regoliths under reduced gravity can be investigated using parabolic flights (Colwell et al., 2008), drop towers or Atwood machines (Goldman \& Umbanhowar, 2008). Atwood machines accelerate the target at a fraction of Earth's gravitational acceleration by using a balancing weight while the impactor falls freely in Earth's gravity. This method does do not allow the impact of large masses in the $100 \mathrm{~kg}$ range as would be expected for a microsatellite sized spacecraft.

To test full-scale landing impact tests of spacecraft penetrators for subsurface exploration (Paton et al., 2012b, 2015) and support interpretation of the Huygens penetrometer measurements on Titan (Zarnecki et al., 2005) a test rig was built at the Open University in the UK. The design was constrained by experimental requirements and the restricted floor space of the laboratory. To prevent the granular analogue regolith targets falling out of their containers it was necessary to have an apparatus where the impact was normal to the acceleration due to gravity. This discounted an arrangement involving inclined planes and pendulums.

Scale models were also discounted as the instruments may have caused the models to be too heavy and interaction with the analogue regolith would have been difficult to interpret. A modified Atwood machine was chosen using balanced weights connected in a loop to reduce the effective acceleration on the spacecraft during impact but not the target. Importantly it allowed impacts and thermal measurements of fragile dusty targets that may represent the surface of a regolith in a low gravity environment, without the spacecraft compressing the target under its own weight after coming to rest (Paton et al., 2012b).

The test rig, shown in figure 3, enabled Earth's gravity to be balanced out and to simulate the rigid body of the spacecraft. The spacecraft mass, $m$ (about $100 \mathrm{~kg}$ ) was divided in two $\left(m_{1}\right.$ and $\left.m_{2}\right)$ for simulating a landing in the low gravitational environment on an asteroid, placing each half on a chain that runs over an arrangement of cogs. For later simulations of impacts on Titan the total mass was reduced, to allow higher impact speeds, with the 
front mass being out of balance with the back to simulate the gravitational acceleration on Titan. The test rig consists of two sets of chain loops running in parallel that enable a simulated spacecraft base plate and the weights to be mounted on a cross-beam between the chains at the front of the rig. At the back of the rig there is a similar arrangement with a cross-beam with weights mounted on it. Each end of the cross-beam is mounted on slider type trolleys each side of the test rig. The trollies run on vertically mounted rails and the chains are attached to the top and underside of the trollies.

To accelerate the system up to impact speed, an accelerating mass $\left(m_{3}\right)$ is attached to the back mass by a cable running over a roller. The cable is guarded against slipping off the roller by two large steel discs attached to the side of the roller. The accelerating mass $m_{3}$ is disengaged from the system by impacting the ground just before the penetrometer reaches its target. The penetrometer is connected to a base plate, which in turn is attached under the front cross-beam and contributes to the front mass, $m_{1}$. Upon impact, forces are directed in a vertical direction.

Table 1: Masses on the test rig

\begin{tabular}{lll}
\hline \multirow{2}{*}{ Mass } & Description & Mass $(\mathrm{kg})$ \\
\hline$m_{1}$ & Front mass including weights $(25 \mathrm{~kg})$ & 53 \\
& Front cross-beam $(6 \mathrm{~kg})$, baseplate $(9 \mathrm{~kg})$ & \\
& Friction balance $(11 \mathrm{~kg})$, other $(2 \mathrm{~kg})$ & \\
$m_{2}$ & Back mass including weights $(40 \mathrm{~kg})$ & 46 \\
& Cross-beam $(6 \mathrm{~kg})$ & 27 \\
$m_{3}$ & Accelerating masses & 4 \\
$m_{4}$ & Chain &
\end{tabular}

Before the test rig can perform a drop the accelerating mass, $m_{3}$ needs to be raised by means of a winding handle fixed to one of the rig's shafts. Raising the accelerating mass also has the effect of raising the penetrometer and associated mass, $m_{1}$ and lowering the balancing mass on and including the back cross-beam, $m_{2}$. After this had been done the operation of the test rig can be separated into four distinct phases or stages as listed below. The stages (a) to (d) listed below correspond to the stages (a) to (d) shown in figure 4 .

(a) Once the front weight has been raised the accelerating mass is then 


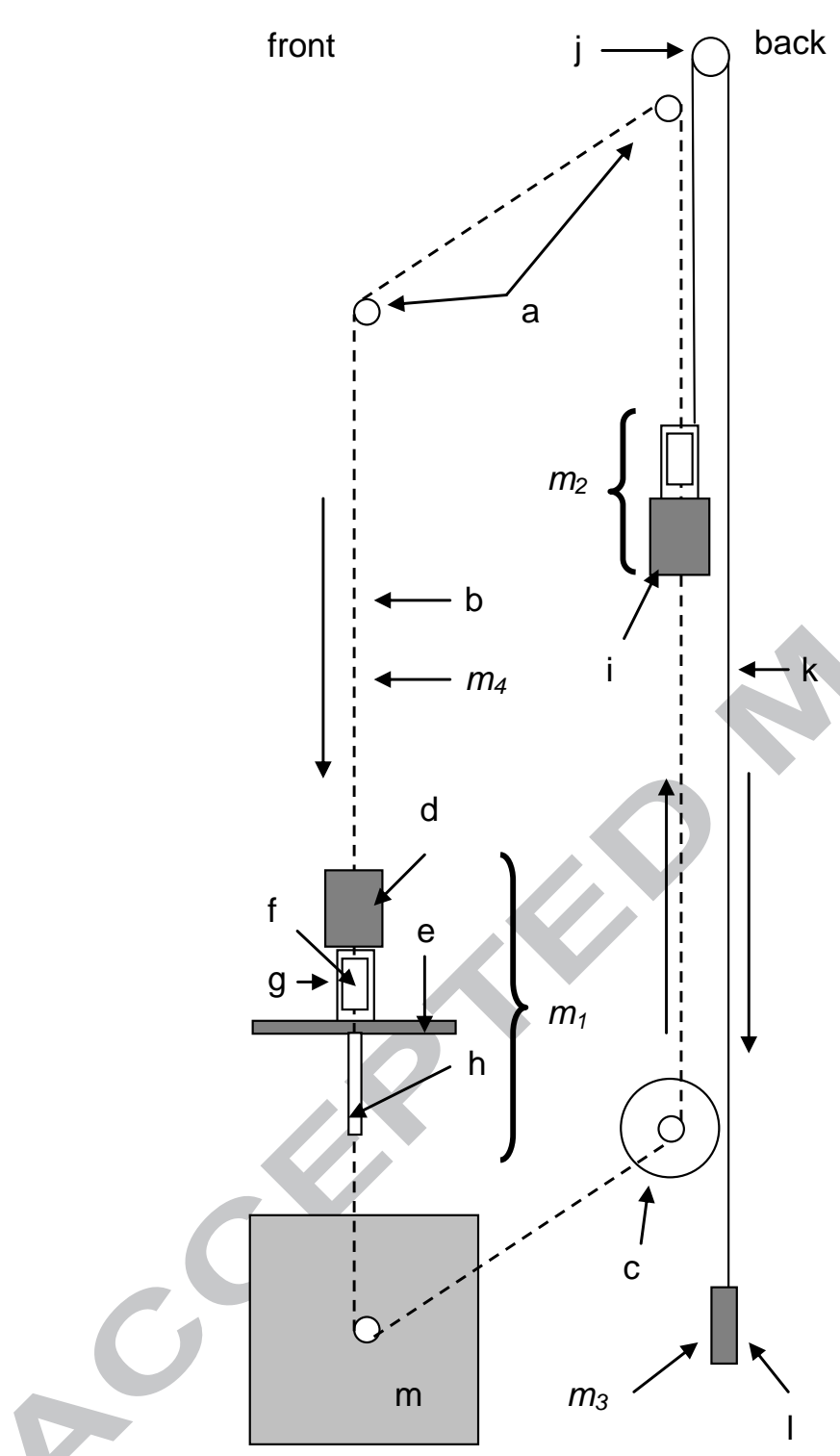

(a) Schematic side-view

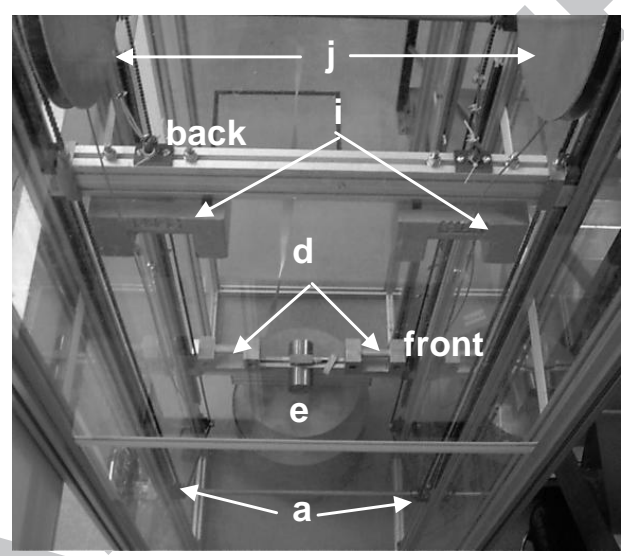

(b) rig viewed from above and behind

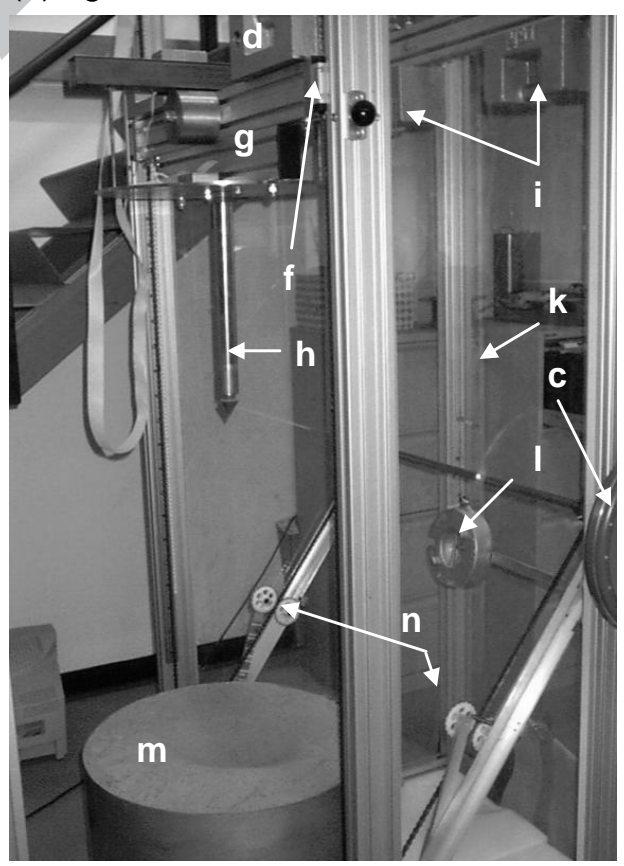

(c) rig viewed from the side

Figure 3: Laboratory simulation of a spacecraft landing using a test rig. Figure reproduced from Paton et al. (2015). The schematic on the left shows the principle of operation using a loop to simulate the inertia of a rigid body and balancing weights to balance out Earth's gravity. On the right is the rig in the laboratory with a large penetrometer attached to the simulated spacecraft base. (a) are the cogs, (b) chain, (c) operator's winding handle, (d) front weights (e) base plate, (f) trolley, (g) front cross-beam structure, (h) penetrometer, (i) back weights $(\mathrm{j})$ accelerating mass roller $(\mathrm{k})$ accelerating mass cable (l) accelerating weight (m) NEO analogue material and (n) chain guides, (m1) front mass, (m2) back mass, (m3) accelerating mass, (m4) chain mass. 

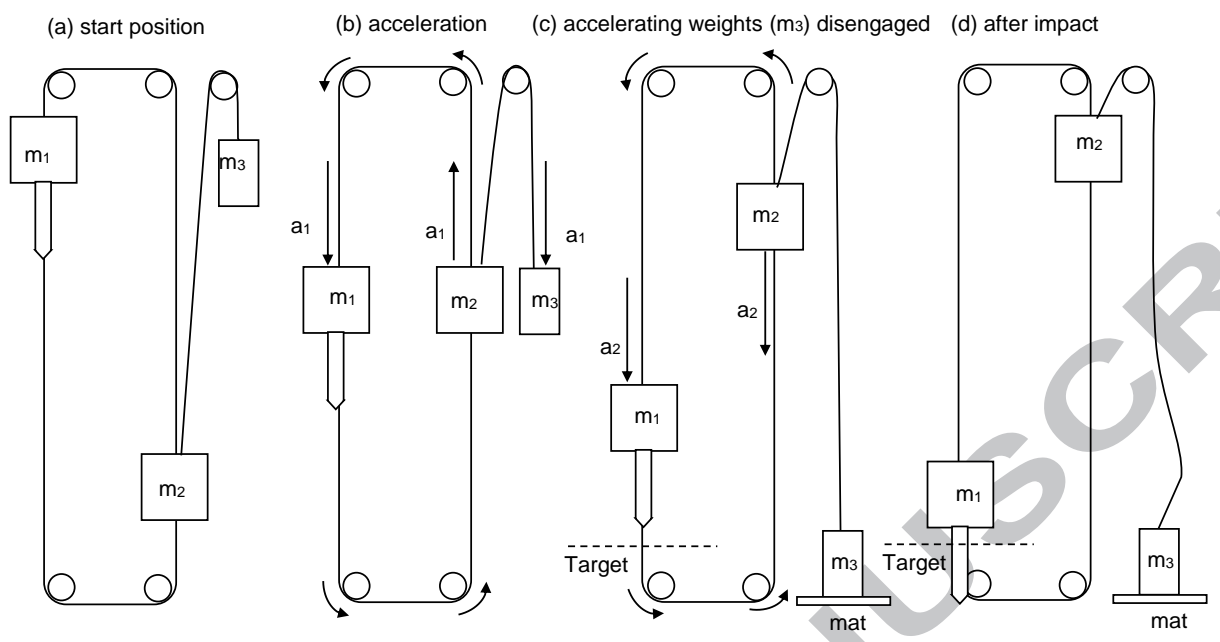

Figure 4: The four configurations of the test rig during a drop.

released by pulling out the winding wheel. The accelerating mass can be released from heights of up to $1.45 \mathrm{~m}$ above the lip of the steel bucket used for holding the target. This is the release phase and is more or less instantaneous.

(b) Gravity accelerates the accelerating mass, $m_{3}$, causing the front mass $\left(m_{1}\right)$ to accelerate downwards and the back mass $\left(m_{2}\right)$ to accelerate upwards. This is the acceleration phase.

(c) The coasting phase begins when the accelerating mass $\left(m_{3}\right)$ hits the ground and is disengaged from the moving front and back masses. When the front and back masses are in balance $\left(m_{1}=m_{2}\right)$ for simulating a landing on an asteroid the coasting phase will be a period of constant speed equal to the speed attained at the end of the acceleration phase. If the front mass is larger than the back mass $\left(m_{1}>m_{2}\right)$ there will be a downward acceleration. In this way the acceleration during penetration can be controlled.

(d) The impact phase begins when the penetrometer tip touches the target.

\subsection{Instrumentation and tools on the test rig}

\subsubsection{Shaft Encoder}

The shaft encoder is attached to a cog on the test rig as shown in figure 5. Inside the shaft encoder a light sensitive diode detects pulses from a source behind a slotted rotating disk, which can then be output to an ADC. The shaft encoder produces 256 pulses per revolution. These pulses can 


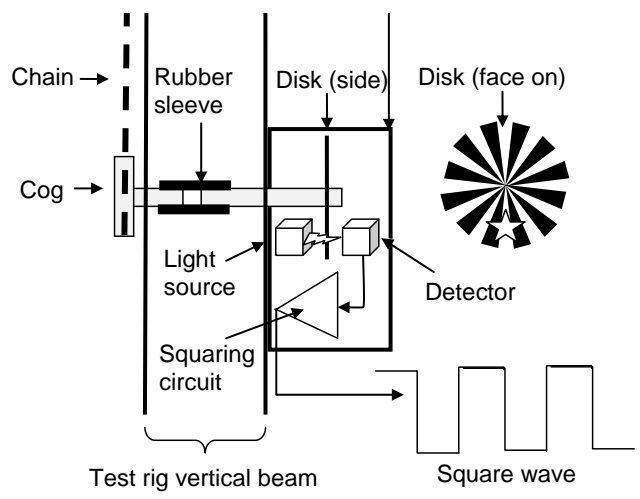

Figure 5: Schematic of the shaft encoder mounted on the test rig.

be electronically combined by the shaft encoder to reduce the number of output pulses. A slider switch next to the shaft encoder is used to select the shaft encoder pulse output $(256,128,64,32,16,8$ and 4 pulses per revolution). Each pulse is output in volts as a square wave as shown in figure 5. The pulses can be counted to determine the angle rotated by the cog and hence the distance travelled by the chain over the cog. The speed of the chain can be calculated by dividing the pulse distance by the period of the pulse. As well as a diagnostic tool the shaft encoder provides important information for penetrometry experiments such as the impact point, impact speed, penetration depth and speed with depth. The impact point of the penetrometer can be determined by counting the pulses.

The maximum speed at which the shaft encoder can operate is ultimately fixed by its mechanical limits. An rpm rating, given by the manufacturer, varies between 300 and 2000 depending on the amount of drag applied. Drag can be applied to the shaft encoder to dampen any vibrations that may feed into the LED collimator. The lower rating is for when maximum drag is applied. The upper limit is for when the shaft encoder is spinning freely. Assuming the distance travelled by the rig's chain during one revolution is equal to the pitch of the $\operatorname{cog}(12.7 \mathrm{~mm})$ multiplied by the number of teeth (12), which is $152.4 \mathrm{~mm}$, then the maximum operational speed will vary from $0.76 \mathrm{~m} \mathrm{~s}^{-1}$ to $5.08 \mathrm{~m} \mathrm{~s}^{-1}$ depending on how much drag is applied to the shaft encoder. 


\subsubsection{Test rig accelerometer}

To complement the measurements made by the shaft encoder a single axis accelerometer was bolted to the front bar of the test rig. A single axis accelerometer was chosen because the simulated spacecraft on the test rig primarily moves along one axis.

The accelerometer chosen was a commercially available single axis DC sensitive ADXL150 that is based on Microelectro-mechanical (MEMS) technology. It is suited for measuring slowly-changing and constant accelerations as would be expected on the test rig before impact. The accelerometer can also provide a check on the speed calculated using shaft encoder data by integration of the acceleration measurements during the descent. The impact speed is required for penetrometry modelling as an initial condition.

Impact accelerometers used in the Huygen's SSP accelerometer and the Rosetta lander anchor are based on piezoelectric technology. These types of accelerometers respond best to high frequency (AC) signals. The SSP accelerometer, ACC-I (Zarnecki et al., 1997), is an Endevco 2271 AM20 with a range of $\pm 90 \mathrm{~g}$. The Rosetta lander anchor (Thiel et al., 2003) is an Endevco Isotron 2255B-1 with a range of $\pm 5000 \mathrm{~g}$. The Huygens ACC-I measured a peak deceleration of $18 \mathrm{~g}$ and the acceleration signal was integrated to give a measurement of $4.63 \mathrm{~m} \mathrm{~s}^{-1}$, which compares favourably to impact speed measurements using other instruments on Huygens.

The accelerometer box with its conditioning and amplification circuit is bolted onto the front beam of the test rig. Mounting the circuitry on the rig prevents amplification of any noise from long connecting cables. The accelerometer itself is rigidly connected to the floor of the box with epoxy resin to ensure good mechanical contact with the test rig. The ADXL150 has a range of $\pm 50 \mathrm{~g}$ similar to that for the SSP accelerometer, with a resolution of $10 \mathrm{mg}$. Our accelerometer is DC sensitive as opposed to AC as the motion of the test rig will experience relatively long periods of constant accelerations as it accelerates the penetrometer up to impact speed. The ADXL150 frequency response is adequate for our purposes as it can measure frequencies up to $300 \mathrm{~Hz}$ without any reduction in output.

\subsubsection{Surface contact accelerometer}

An identical accelerometer to that mounted in the test rig was attached to the underside of the base plate and hung down on a length of cable so it impacted the surface at the same time as the tip of the penetrometer touched the surface. The length of cable for the accelerometer was adjusted 
before the test by manually lowering the penetrometer on the test rig until its tip was just touching the surface of the target. The accelerometer is mounted in a metal box with its conditioning electronics as is the main test rig accelerometer. This technique was found to be a reliable method to quickly, i.e. no data processing required, and precisely determine the starting point of the penetrometer impact. An uncertainty of about $\pm 5 \mathrm{~mm}$ was assigned to this technique.

\subsubsection{Tape measure and plastic rod}

A ruled measure was taped on the side of the test rig to allow the measurement of the drop height of the base plate. The zero point was located parallel with the top of the large steel bucket that contained the target. The ruled measure was most useful when selecting a starting position for the drop. For determining the penetration depth of the rod or penetrometer, independently of the shaft encoder or accelerometer, a steel ruler was used. This was required because the surface of the target was below the top of the bucket.

A plastic cylindrical rod measuring $380 \mathrm{~mm}$ in length and with a diameter of $35 \mathrm{~mm}$ was attached to the underside of the base plate. This was used during drop tests to decelerate the base plate and prevent it from impacting the target which may have caused damaging shocks to the test rig as a consequence of its large surface area.

\subsubsection{Experimental impact penetrometer (X-PEN) and the Huygens pen- etrometer $(A C C-E)$}

The experimental cone penetrometer (X-PEN), used in this work, is shown in figure 6. Its dimensions are based on the Cone Penetration Testing (CPT) design used in civil engineering applications. X-PEN has previously been used to investigate measurements in analogue planetary regoliths by Paton et al. (2015). The penetrometer is $40 \mathrm{~cm}$ in length, $3.8 \mathrm{~cm}$ in diameter and the conical tip has a $60^{\circ}$ apex angle. A load cell is mounted behind the tip. The ACC-E penetrometer (Lorenz et al., 1994) shown in figure 7 has a hemispherical tip with a diameter of $16 \mathrm{~mm}$ and uses a piezoelectric mounted behind the tip to sense the force on the tip. It was used on the Huygens mission to measure the mechanical properties of Titan's surface (Zarnecki et al., 2005). ACC-E was part of the Huygens Surface Science Package that was designed at the time for a largely unknown Titan surface. 


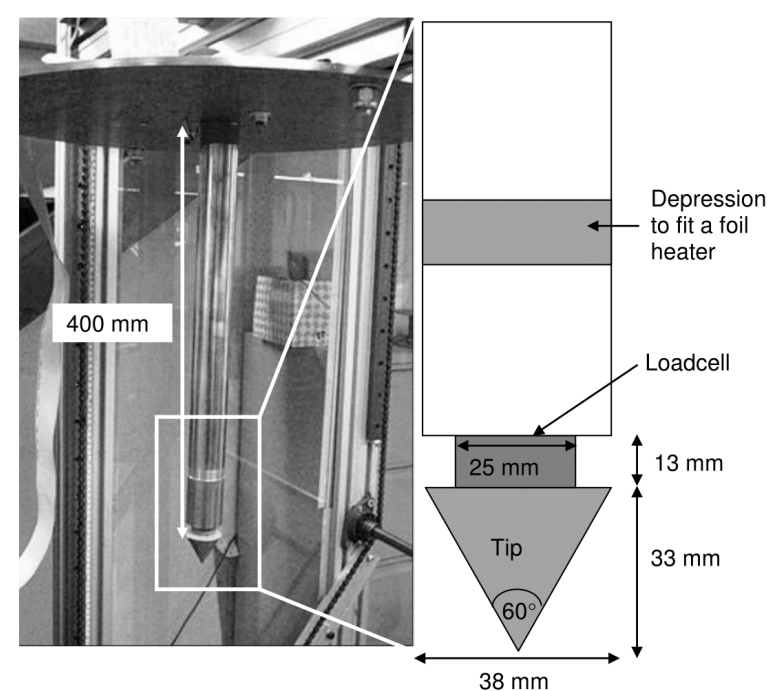

Figure 6: Experimental penetrometer attached to the test rig base plate. Figure reproduced from Paton et al. (2015). Components of the test rig can be seen in the background: the chain passing over a cog (lower right); the front beam (top). The experimental penetrometer is attached to the underside of the base plate. The depression in the shaft of the penetrometer was intended to contain a film heater for making thermal measurements. Thermal measurements using a thermal probe based on this design can be found in Paton et al. (2012b). 

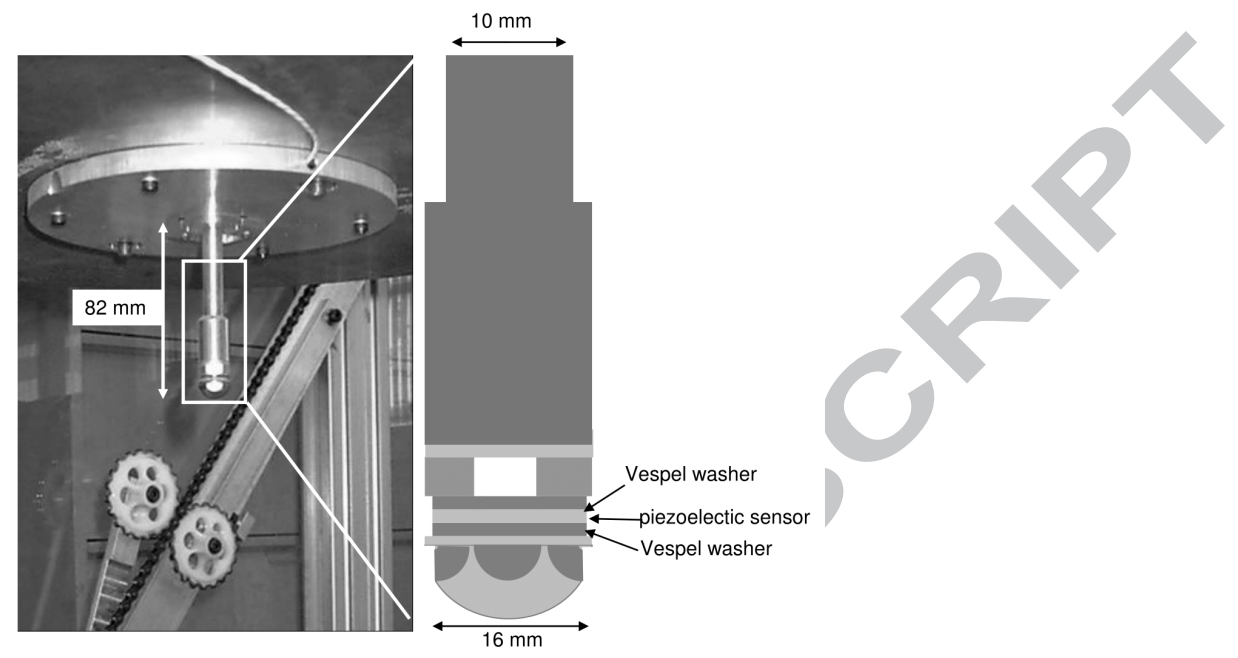

Figure 7: Huygens ACC-E penetrometer and adaptor plate attached to the underside of the test rig base plate. The figure is reproduced from Paton et al. (2015). In the background is a section of the chain. ACC-E was used in the results section to compare with the Huygens flight data from Titan.

\subsection{Instrument errors}

Measurements can suffer from systematic as well as random errors, dependent on the instrument design. Determining position only using the shaft encoder will be problematic because there is ambiguity in the direction of travel and the precise position of the test rig can only be known during the transition between a high and low state of the output signal. Measuring speed precisely using the shaft encoder will depend on the sample rate and the width, in terms of distance, of the square wave output. A small wavelength requires a higher sampling rate and vice versa. Therefore for measuring speed the shaft encoder needs to be configured so each high and low state corresponds to a large distance and so could be sampled adequately when the rig was travelling at high speed. This is in contrast to measuring distance where a smaller wavelength is ideally required for precise measurements of position.

When using the accelerometer, uncertainties in distance and speed measurements originate from structural vibrations inherent in the mechanical operation of the test rig making the signal appear noisy. Similar noisy signals are apparent in other guide-rail apparatus, e.g. Lorenz et al. (2015). Integrating the oscillations in the accelerometer, data which vary in ampli- 
tude and frequency, indicates an uncertainty of about $0.04 \mathrm{~m} \mathrm{~s}^{-1}$ at an impact speed of $2 \mathrm{~m} \mathrm{~s}^{-1}$ and integrating again results in an oscillation in the vertical position of the baseplate of about $1 \mathrm{~mm}$. Small changes in the bias were also observed causing the accumulation of uncertainties.

The uncertainties with and without the oscillations of the chain taken into account are shown in table 2 .

Table 2: Accelerometer and shaft encoder measurement uncertainties with and without vibration of the rig taken into account. The distance travelled was $190 \mathrm{~cm}$ and the impact speed was $2 \mathrm{~m} \mathrm{~s}^{-1}$.

\begin{tabular}{llll}
\hline $\begin{array}{l}\text { Instrument } \\
\text { Instrument }\end{array}$ & $\begin{array}{l}\text { Distance } \\
\text { uncertainty } \\
(\mathrm{cm})\end{array}$ & $\begin{array}{l}\text { Speed } \\
\text { uncertainty } \\
(\mathrm{cm} \mathrm{s})\end{array}$ & $\begin{array}{l}\text { Acceleration } \\
\text { uncertainty } \\
\left(\mathrm{m} \mathrm{s}^{-2}\right)\end{array}$ \\
\hline Accelerometer & 1.6 & 0.8 & 0.004 \\
Shaft encoder & 2.0 & 2.1 & - \\
Accelerometer (chain oscillations) & 5.4 & 1.9 & 5 \\
Shaft encoder (chain oscillations) & 6.0 & 3.8 & - \\
Ruler & 0.1 & - & - \\
\hline
\end{tabular}

\subsection{Target properties}

Table 3 lists the target properties used during the experiments. The finegrained materials (limestone powder, iron powder and sand) were used to model layered regolith and investigate the response of the penetrometer to such layering. The coarser-grained gravel was used to determine how well the penetrometer (X-PEN) could measure particle size and mass.

Figure 8 illustrates the layered targets used in our experiments. The tip of the penetrometer is included so the relative scale can be seen. Figure 9 shows close-up images of the fine-grained materials used for the penetrometry experiments in layered materials. Figure 10 shows the gravel material used for experiments to detect particle properties.

\section{A numerical approach to mechanical modelling}

\subsection{Forces on the test rig}

The motion of the test rig can be described approximately using Newton's second law. The resultant force on the test rig equals the weight of the front 
Table 3: Surface analogue material properties. The gravel materials were used for particle detection experiments and investigating the penetrometer data from Huygens. The rows contain the following information: average diameter $(d)$, angle of repose $(\theta)$, sphericity $(Y)$, roundness $(R)$, bulk density $\left(\rho_{0}\right)$, solid density $\left(\rho_{s}\right)$, cohesion $(c)$, friction angle $(\phi)$.

\begin{tabular}{rrrrrrrrr}
\hline & Lime & Iron & Sand & Gravel & Gravel & Gravel & Gravel & Gravel \\
\hline $\mathrm{d}(\mu \mathrm{m})$ & 5 & 95 & 200 & 3640 & 4430 & 6760 & 8960 & 14850 \\
$\theta^{\circ}$ & 50 & 42 & 28 & - & - & - & - & - \\
$Y$ & 0.86 & 0.79 & 0.95 & 0.6 & 0.63 & 0.68 & 0.65 & 0.66 \\
$R$ & 0.25 & 0.16 & 0.55 & - & - & & - & \\
$\rho_{0}\left(\mathrm{~g} \mathrm{~cm}^{-3}\right)$ & 0.94 & 2.71 & 1.29 & 1.42 & 1.85 & 1.67 & 1.56 & 1.56 \\
$\rho_{S}\left(\mathrm{~g} \mathrm{~cm}^{-3}\right)$ & 2.6 & 7.9 & 2.5 & 2.5 & 2.5 & 2.5 & 2.5 & 2.5 \\
$\mathrm{c}(\mathrm{kPa})$ & 8 & 0 & 0 & 0 & 0 & 0 & 0 & 0 \\
$\phi^{\circ}$ & 35 & 43 & 38 & $36-42$ & $36-42$ & $36-42$ & $36-42$ & $36-42$ \\
\hline
\end{tabular}
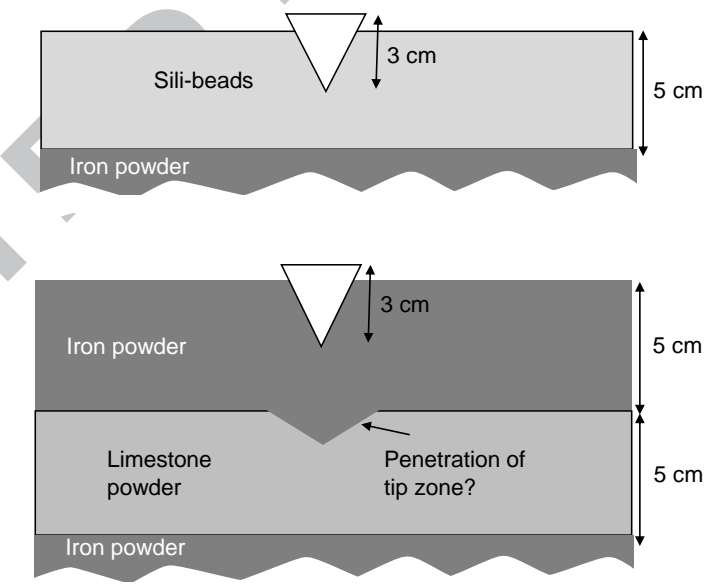

Figure 8: Schematic of layered material targets used for layer detection and investigation experiments. The materials are given in table 3 except Sili-beads. Sili-beads are glass spheres with a radius of $2 \mathrm{~mm}$ and a solid density of $2.5 \mathrm{~g} \mathrm{~cm}^{-3}$. The upper and lower schematics correspond to X-PEN measurements shown in figures 18 (a) and (b) respectively. 

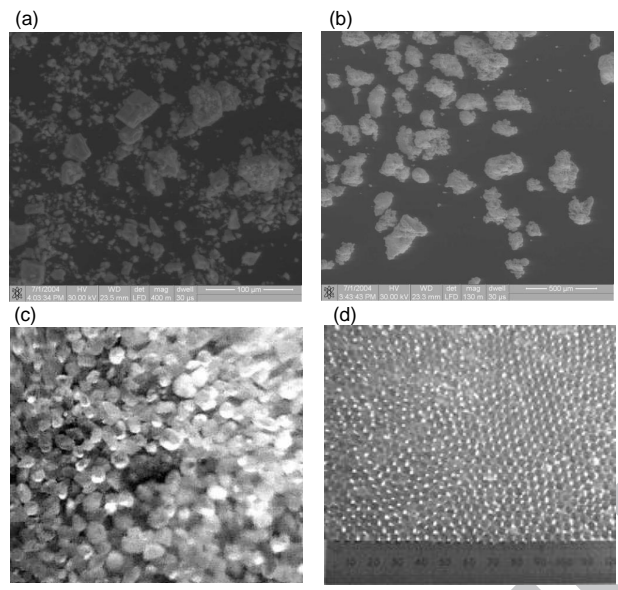

Figure 9: Fine grained materials used to construct the layered targets. Figure (a) shows a close up of limestone powder grains, (b) shows iron powder grains, (c) shows sand grains and (d) shows $4 \mathrm{~mm}$ diameter sili-beads.

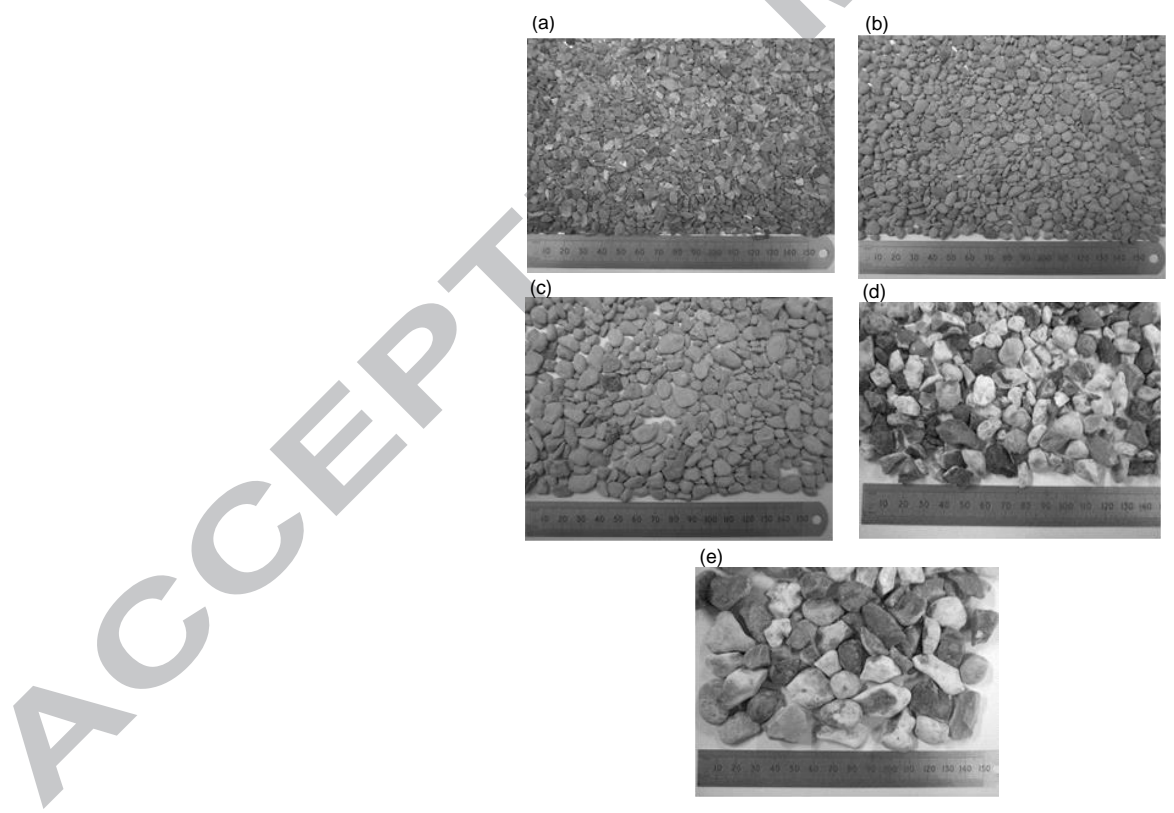

Figure 10: Coarse grained materials used for particle size and mass detection tests. The materials (a) to (e) are gravel targets with increasing average particle diameters and correspond to the materials in table 3 . 
mass $\left(m_{1} g\right)$, minus the weight of the back mass $\left(m_{2} g\right)$ plus the force of the accelerating mass $\left(m_{3} g\right)$ minus the friction, $F_{f}$. The inertial mass of the test rig includes the mass on and including the front cross-beam, $m_{1}$, plus the mass on and including the back cross-beam, $m_{2}$, plus the mass of the accelerating weights, $m_{3}$, plus the mass of the chain, $m_{4}$. The test rig's acceleration can then be expressed as in equation 1.

$$
\frac{d u}{d t}=\frac{\left(m_{1}-m_{2}\right) g+m_{3} g \pm F_{f}}{\left(m_{1}+m_{2}+m_{3}+m_{4}\right)}
$$

where $g$ is acceleration due to Earth's gravity, $F_{f}$ is the frictional force. Friction within the system is partly counterbalanced with an extra weight placed on the front beam. The \pm sign in equation 1 is to indicate that friction is present in both directions. With friction set to zero in equation 2 and the acceleration substituted into the equation $v^{2}=2 a S$ where $a$ is the acceleration and $S$ is the acceleration distance, the predicted impact velocities will be $2.2 \mathrm{~m} \mathrm{~s}^{-1}$ for the rig configured to simulate landings under zero-g, i.e. with masses as given in Table 1.

The acceleration of the test rig during the impact phase, for simulating impacts on a body with a significant acceleration due to gravity, can be set by varying either the mass on the front beam or the mass on the back beam or both. This allows the simulation of a spacecraft landing on any Solar System body with an acceleration due to gravity lower than that of Earth, e.g., the Moon, Mars, Titan or an asteroid. This effective acceleration due to gravity can be calculated as follows.

$$
g_{\text {eff }}=g \frac{m_{1}-m_{2}-F_{f} / m_{\text {total }}}{m_{1}+m_{2}+m_{4}}
$$

where $g$ is the acceleration due to gravity on Earth. The effective gravitational acceleration can be tuned for any planetary body in the Solar System with a $g_{\text {eff }}<g$ by varying $m_{1}$ and $m_{2}$. The coasting phase, after the accelerating weights disengage and before the impact of the base plate is $0.45 \mathrm{~m}$ in length.

$$
F_{\text {rig }}=F_{m 1}-F_{m 2}-F_{f}-F_{p e n}
$$

where $F_{\text {pen }}$ is the force on the penetrometer. To analyse the response of the test rig chains to changes in tension when the weights are released, when the accelerating weights disengage and during the impact, a lumped 
mass-spring model is used. Equations 4 and 5 below model the mechanical response of the test rig during the acceleration phase (stage b in figure 4) and the impact phase (stage $\mathrm{d}$ ).

$$
\begin{aligned}
& \sum_{i=3}^{i=0} m_{i} \ddot{z}_{i}+k_{i}\left[z_{i}-z_{i+1}\right]+c\left[\dot{z}_{i}-\dot{z}_{i+1}\right]=F_{m 1}-F_{m 2}+F_{m 3}+F_{f} \\
& \sum_{i=2}^{i=0} m_{i} \ddot{z}_{i}+k_{i}\left[z_{i}-z_{i+1}\right]+c\left[\dot{z}_{i}-\dot{z}_{i+1}\right]=F_{m 1}-F_{m 2}+F_{f}+F_{p e n}
\end{aligned}
$$

where $i$ represents a component of the test rig model, i.e. the mass of the tip $(i=0)$, the front mass $(i=1)$, the back mass $(i=2)$ and the accelerating weight $(i=3)$. The masses $m_{0}$ to $m_{3}$, the spring, $k_{0}$ to $k_{2}$, and damping coefficients, $c_{0}$ to $c_{2}$ are shown in figure 11 and described in the caption. Figure 12 shows results from the test rig mechanical model compared to what would be expected landing on an asteroid or comet by a rigid spacecraft. For the rigid spacecraft impact, figure 12 (a) and (b), the acceleration of the spacecraft and the force on the penetrometer follow each other closely. For the impact of the test rig on the same target there is some oscillation in the acceleration of the front mass due to the elasticity of the chain. This is caused by changes in tension of the chain during different phases of operation, i.e. acceleration phase, impact of accelerating weights and impact of the penetrometer. The oscillation in the acceleration of the front mass does not impact the penetrometer measurement because the force on the penetrometer is dependent on the depth of penetration.

\subsection{Penetrometer model}

\subsubsection{Background on the penetrometer model}

Lorenz et al (1994) conducted impact tests of a hemispherical tipped penetrometer (ACC-E) in gravel materials. The tests produced force profiles featuring a sequence of spikes. It was found the interval between the peaks increased with increasing particle size. Lorenz et al. (1994) proposed that the magnitude of peaks generated from impacts into gravel material corresponds to particle mass and thus by determining mass and size from penetrometry data the solid density of the particles could be estimated.

Paton et al. (2008) conducted free-fall impacts into gravel targets with ACC-E and found the number of peaks in the penetrometry data, over the 


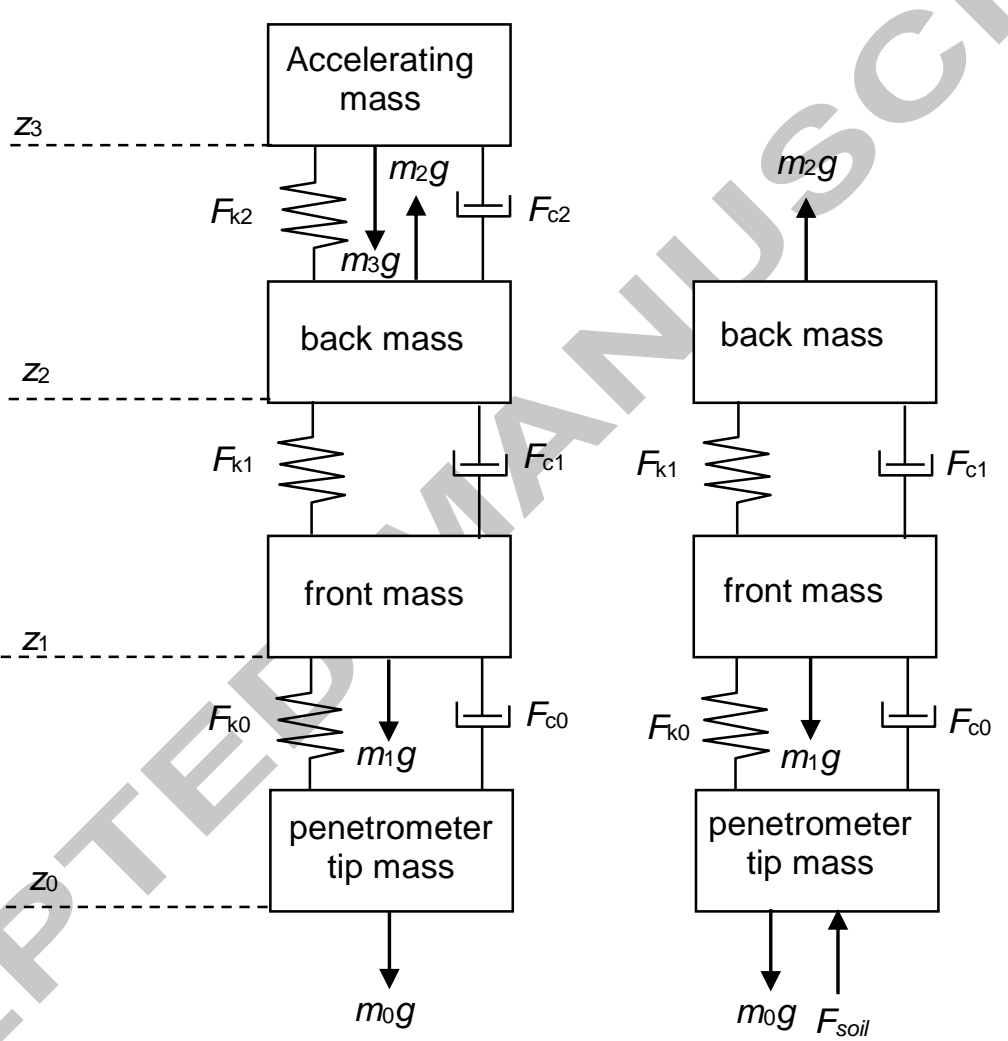

Figure 11: A lumped mass-spring model of the test rig and penetrometer before and after the accelerating weights are disengaged from the system. The masses $m_{0}, m_{1}, m_{2}, m_{3}$ represent the penetrometer tip, front mass, back mass and accelerating weights respectively. The mass of the chain, i.e. $m_{4}$, is not required for the model. Masses $m_{1}$ to $m_{3}$ are listed in table 1. The mass of the penetrometer tip, $m_{0}$, is $0.1 \mathrm{~kg}$. The vertical displacement of the masses from their equilibrium position is represented by the values $z_{0}$ to $z_{3}$. The spring and damping coefficients for the penetrometer sensor, chain and cable are represented by $k_{0}$ to $k_{2}$ and $c_{0}$ to $c_{2}$. 

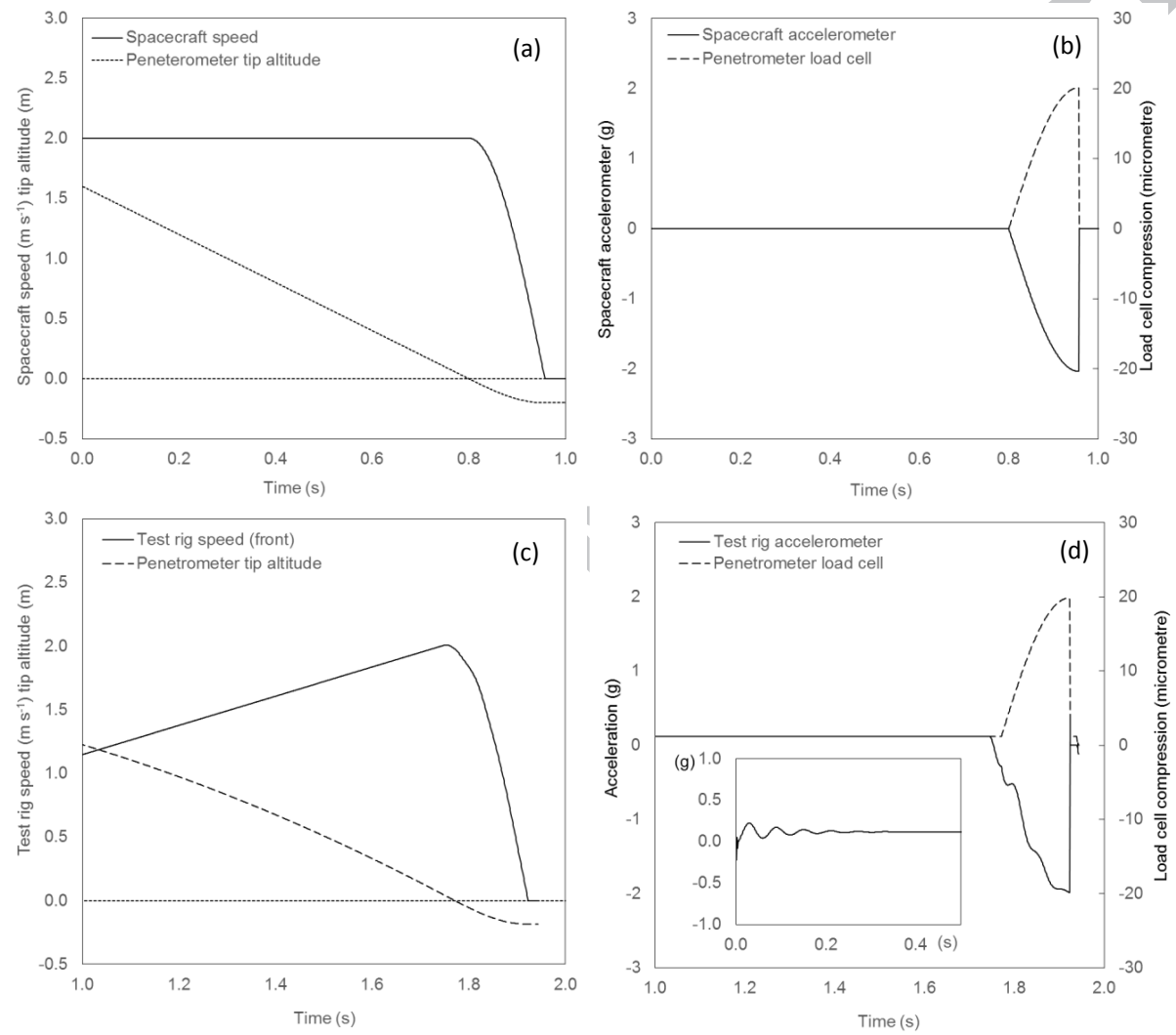

Figure 12: Dynamics of a rigid spacecraft fitted with a $40 \mathrm{~cm}$ long penetrometer impacting a regolith on an asteroid compared with the dynamics of a simulated spacecraft plus penetrometer using the test rig. 
penetration depth, decreased with increasing average particle diameter and the magnitude of the peaks increased with the average particle mass. It was found that for particles between $4 \mathrm{~mm}$ and $7 \mathrm{~mm}$ in diameter the solid density of the target material could be estimated to an accuracy of about $10 \%$ by fitting a physically based empirical equation to the data. This was dependent on a statistically significant amount of impacts being measured by ACC-E.

It was noted by Paton et al. (2008) that for single particle impacts on a hemispherical tip the resulting signal is ambiguous and suggested that a conical tip would help resolve this issue explained as follows. The surface of a hemispherical tip becomes parallel to the direction of motion of the penetrometer towards its edge. A particle impacting a point near the edge of a hemispherical tip would produce a similar force normal to the sensor than that of a smaller particle impacting the centre of the tip. With a conical tip this effect of the surface orientation is largely removed although the decreasing radius of curvature of the surface towards the tip of a cone will have some effect (see equation A.1).

Paton et al. (2012a) used a 3D Discrete Element Model (DEM) together with a numerical mechanical model of ACC-E to further investigate penetrometer design issues and interpret the signal returned by the Huygens penetrometer from Titan. Results from the modelling work suggest that the unprocessed Huygens penetrometer signal is consistent with an impact into granular material whose average particle diameter is between 6 and $8 \mathrm{~mm}$. This is similar, although perhaps slightly larger, than particles identified in images taken after the Huygens landing. In the images Keller et al. (2008) identified particles down to a diameter of $3 \mathrm{~mm}$ with a portion of the surface covered in particles having a diameter of around $5 \mathrm{~mm}$.

It was found by Paton et al. (2012a) that for large particles, comparable to the size of the tip, a combination of a high probability of hitting the side of the tip, which is less sensitive, and the prevalence of small peaks from electronic noise (increasingly present between the less frequent peaks from impacts of large particles) significantly limited the range of particle sizes that the penetrometer could measure easily. The model developed by Paton et al. (2012a) only considered the force on the tip from impacting particles (and tip-particle friction during the impact) and not forces transmitted through the particles from shearing of material to the side, i.e. a frictionless material. 


\subsubsection{Adapting the model for a cone tipped penetrometer}

The mechanism of particle detection, assumed for a cone-tipped penetrometer and target, is shown in figure 13. The peaks are generated from the exchange of momentum from particles impacting the tip as modelled by Paton et al. (2012a). As the penetrometer travels pushes deeper into the target particles interactions become more important as the weight above the tip increases, e.g. see Paton et al. (2015). These forces are transferred to the tip which is represented in figure 13 by the undulating background force that increases with depth.

Two simple modifications to the model enable the computer simulation of a cone-tipped penetrometer passing through a granular material. The model, which can be found described in full in the appendix, was updated by modifying the Hertz circular contact area between two spheres, which was assumed for modelling of spherical particles impacting a hemispherical tip. A spherical particle impacting a cone will produce an elliptical contact area (Popov, 2010) which is represented by the effect radius of curvature as $\left(r_{1} r_{2}\right)^{0.5}$ (See equation A.3). Another modification was to include the force from a shearing plane that develops in granular materials when penetrated. The force is modelled using equation 6 .

$$
F_{s}=N_{q} \rho g z
$$

where $\rho$ is the bulk density, $z$ is the depth of the tip of the cone, $g$ is the acceleration due to gravity and,

$$
N_{q}=1.0584 e^{6.1679 \tan \phi}
$$

where $\phi$ is the effective friction angle of the material. The implementation of this force in the model is described mathematically in the appendix.

Figure 14 shows an example of the model output for an impact into gravel with particles sizes smaller than the tip and for gravel with particles comparable in size to the tip. The force from the target with the smaller particles increases with depth as would be expected for a penetrometer shearing material to the side. The force from the target with the larger particles is lower and the peaks are much larger. This is because large voids in the target, in comparison to the tip, allow the penetrometer to pass without having to always mobilise the surrounding particles. 


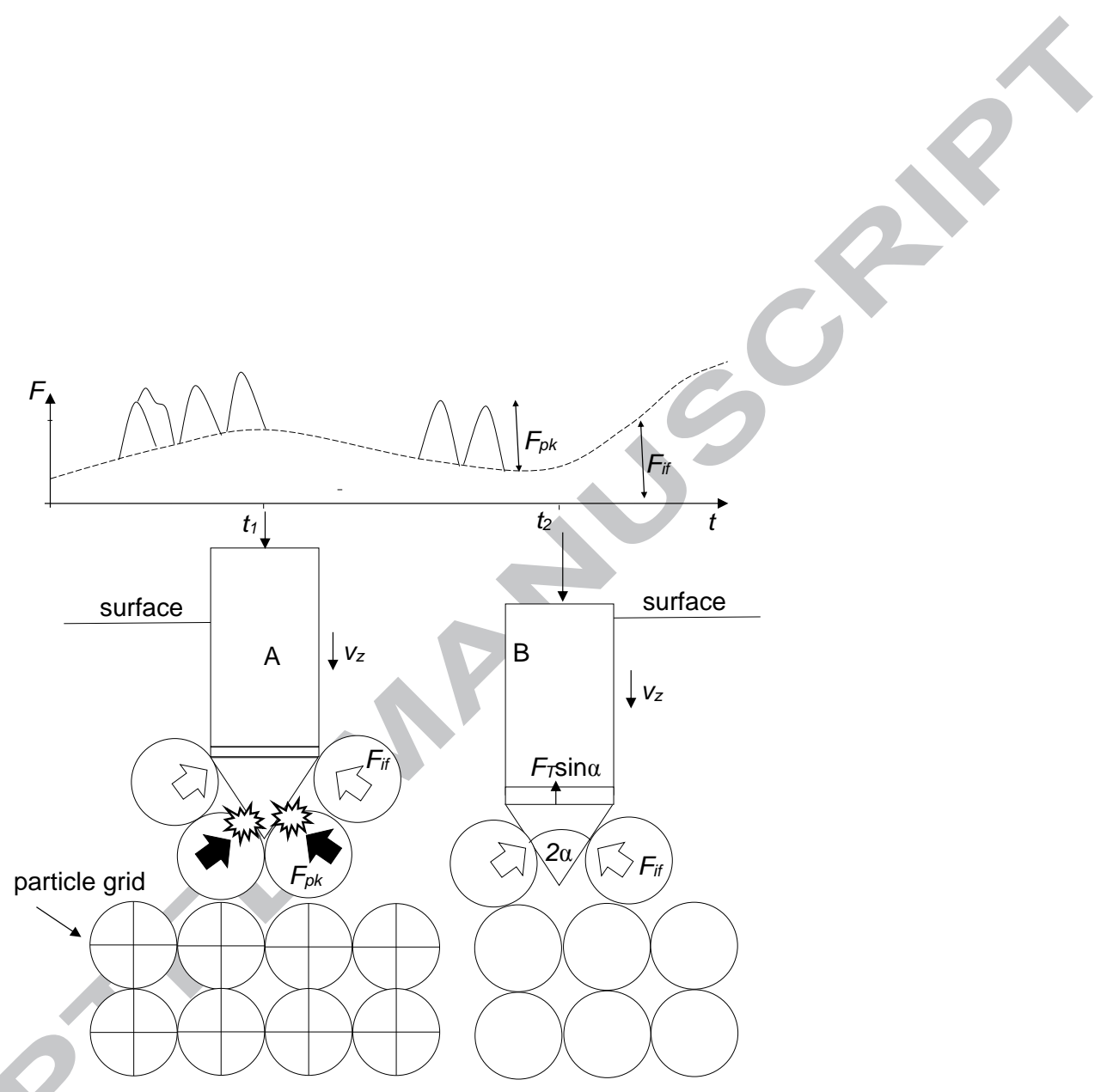

Figure 13: Model of particle-penetrometer interaction adapted from Paton et al. (2012a) for a cone penetrometer. The particles with black arrows are impacting the tip. The white arrows indicate those which are in contact and sliding along the tip. 


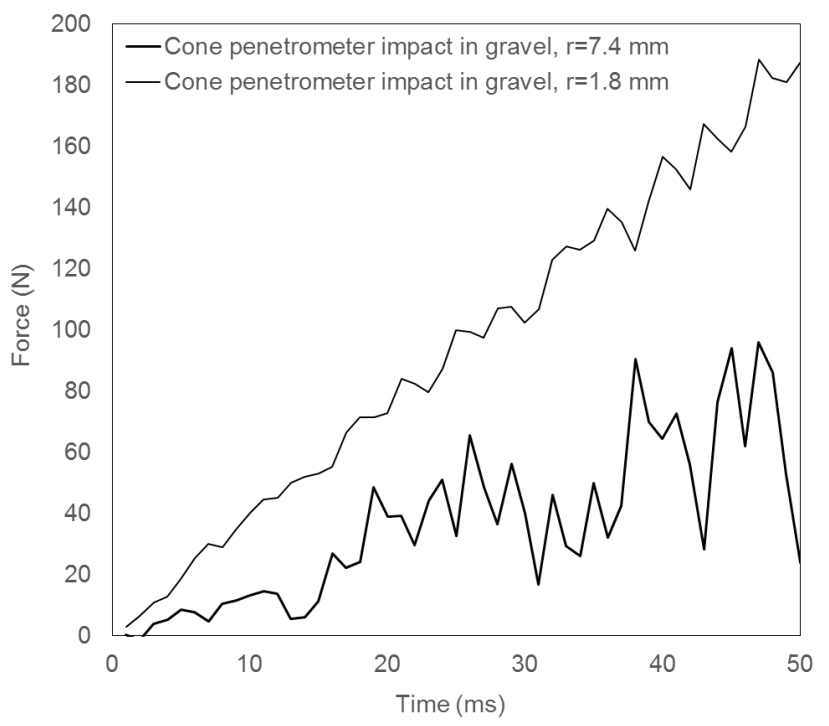

Figure 14: Output from the computer model of a cone-tipped penetrometer impacting targets with different particle sizes. The particles are modelled as monodisperse spheres. The particle radii in the simulated targets are $1.8 \mathrm{~mm}$ and $7.4 \mathrm{~mm}$ with the number of particles in each target being 400 and 16 respectively.

\section{Results}

Drop tests were made to simulate a spacecraft weighing $100 \mathrm{~kg}$ landing on an asteroid. A sand target was used as an analogue asteroid surface. A large rod, similar in dimensions to X-PEN, and a small penetrometer (ACC-E) attached in turn to the underside of the test rig's base plate. The rebound characteristics of the test rig were assessed with a numerical model.

$\mathrm{X}-\mathrm{PEN}$ was used to make measurements in layered analogue regoliths to assess the ability of the penetrometer to detect layered structures and investigate whether a layered regolith could help explain the penetrometry data from the Huygens mission. Impacts into gravel with X-PEN which has a conical tip and ACC-E which had a hemispherical tip were performed and the results compared to further understand a penetrometer's response to the granular material and for investigating the penetrometry data returned from Titan.

\subsection{Test rig model validation and spacecraft rebound}

To investigate the elastic response of the test rig and the potential of a spacecraft to bounce results from laboratory tests and modelling are com- 
pared in figure 15. The 1-D numerical mechanical model of the test rig used to analyse the results is described in section 4.1. A long rod, with a length of $38 \mathrm{~cm}$ and a radius of $35 \mathrm{~mm}$ (similar dimensions to X-PEN), attached to the baseplate to determine if such a device could help absorb the elastic energy of a landing spacecraft. Figure 15 (c) shows the speed of the test rig from an impact test into sand with the smaller ACC-E penetrometer attached to the baseplate. An effective spring constant of the chain of $3.5 \times 10^{5} \mathrm{~N} \mathrm{~m}^{-1}$ was determined by matching the frequency from the modelled test rig chain to measurements from the test rig's accelerometer and shaft encoder. For comparison the effective spring constant of a Philae landing gear leg is 1.3 $10^{4} \mathrm{~N} \mathrm{~m}^{-1}$ (Hilchenbach, 2004). Table 4 shows the values for the relevant properties used in the model.

In figures 15 (a) and (b) the match between the accelerometer data and the model after impact, i.e. the amplitude of the oscillations, was improved by increasing the damping coefficient from, $c=500$ to $c=1000 \mathrm{~N} \mathrm{~s}^{-1} \mathrm{~m}^{-1}$. This suggests the extra dampening results from the rod impacting the sand target. For these tests no rebound speed was detected from analysing the shaft encoder data although integrating the accelerometer after impact suggests a small rebound speed of perhaps $10 \mathrm{~cm} \mathrm{~s}^{-1}$ in figure 15 (a) and $1 \mathrm{~cm}$ $\mathrm{s}^{-1}$ in 15 (b). For the impact test with the baseplate in figure 15 (c) the test rig model damping coefficient did not need to be adjusted after impact. A rebound speed of $20 \mathrm{~cm} \mathrm{~s}^{-1}$ was estimated from the shaft encoder data by averaging the first peak. The motion of the rig was quickly damped by the power loss in the chain and sliding friction.

The ability of a penetrating device to dampen the vibration and rebound of a landing spacecraft may have implications for managing the rebound of a landing spacecraft on a low gravity world. Future landers on low gravity worlds will have an active landing gear (Chitu et al., 2015) to manage the rebound of a spacecraft from the surface during landing and surface operations such as sample collection. On the other hand the results suggests that a spacecraft impact penetrometer could easily be extracted, for further measurements elsewhere, by designing the spacecraft with sufficient elasticity to bounce.

Figure 16 shows the impact speeds for the test rig determined from the accelerometer and shaft encoder. The accelerometer and shaft encoder derived impact speeds are in good general agreement with each other and the predicted impact speeds. The accelerometer derived impact speed appears to deviate slightly from the model is some cases. This could possibly be due 
Table 4: Input values for test rig mechanical model.

\begin{tabular}{ll}
\hline Component & Value \\
\hline Chain $k\left(\mathrm{~N} \mathrm{~m}^{-1}\right)$ & $3.5 \times 10^{5}$ \\
Chain $c\left(\mathrm{~N} \mathrm{~s}^{-1} \mathrm{~m}^{-1}\right)$ & 500 \\
Chain friction $F_{f}(\mathrm{~N})$ & \pm 120 \\
Strength of target $F_{\text {pen }}\left(\mathrm{kPa} \mathrm{m}^{-1}\right)$ & 1000
\end{tabular}
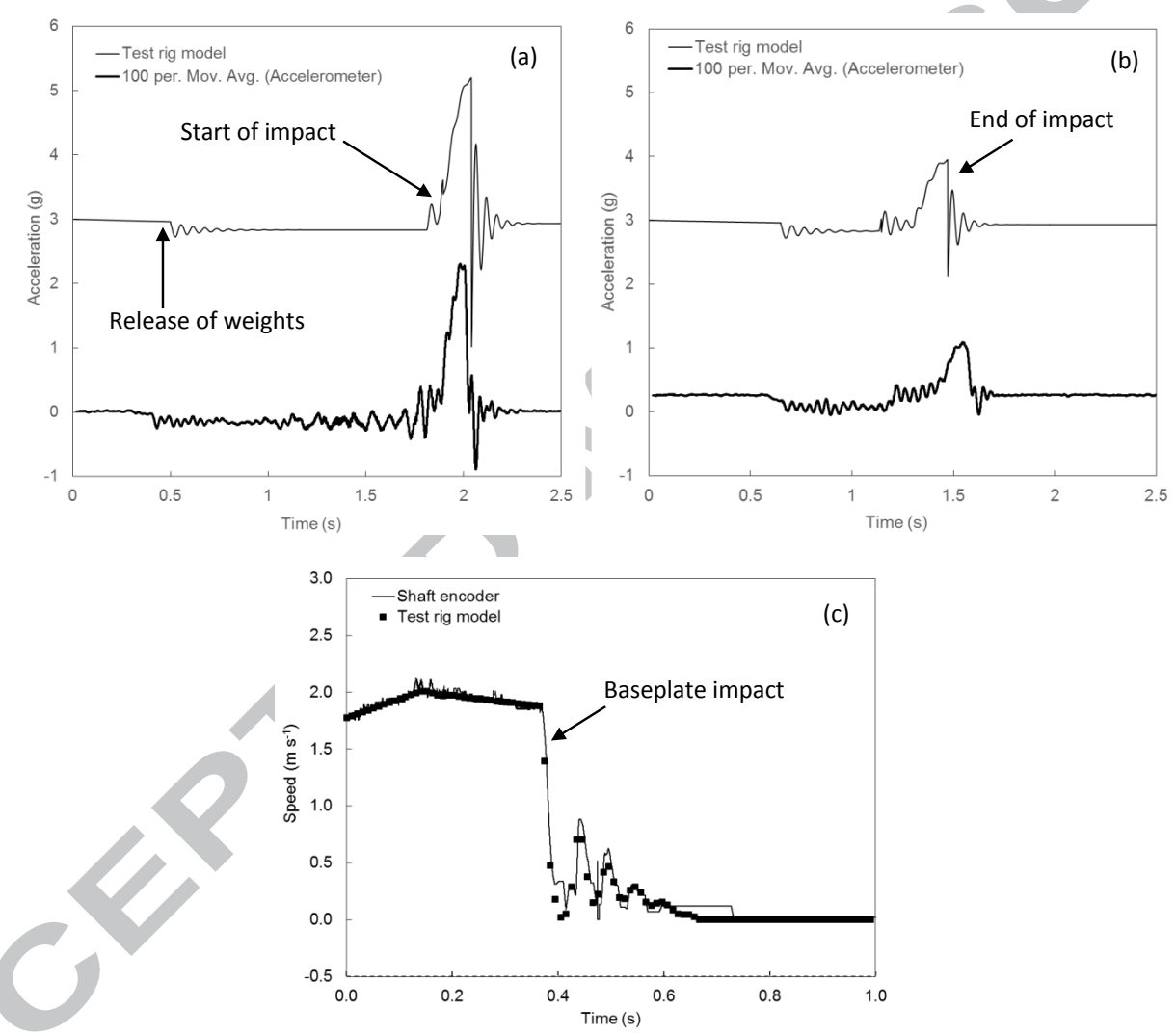

Figure 15: Test rig model compared to laboratory data. (a) and (b) show the acceleration of the test rig with a long rod attached for impacts at speeds of 2.0 and $0.9 \mathrm{~m} \mathrm{~s}^{-1}$ respectively. In (c) the baseplate with the smaller ACC-E impacts the target at a speed of $2 \mathrm{~m} \mathrm{~s}^{-1}$ at around $0.4 \mathrm{~s}$. The speed from the model, after the impact, is shown as a positive quantity to match the speed derived from the shaft encoder which cannot be used to determine the direction of motion. 


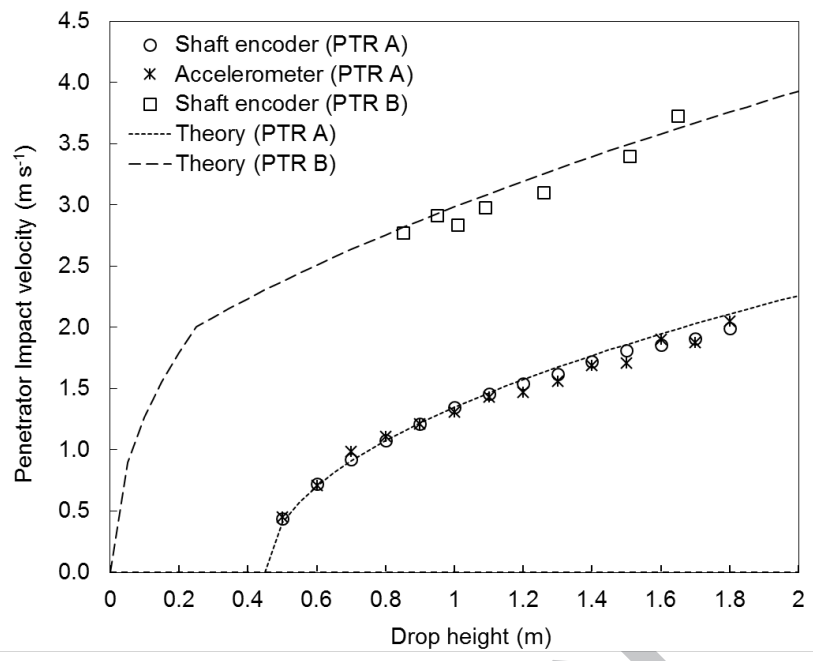

Figure 16: Speed at the end of the acceleration phase compared to theory. The uncertainty in speed is comparable to the marker sizes. PTR A and PTR B refers to the test rig configured for landing on the asteroid and Titan respectively.

to a slight reorientation of the accelerometer after an impact.

\subsection{Penetration of layered analogue regoliths}

A large penetrator X-PEN, similar to that used in Cone Penetration Test (CPT) for civil engineering applications was tested in layered regolith analogues to see how well such structures could be detected and to see if the negative force-depth profile from Huygens could be reproduced. Previous impact tests with the same penetrometer were made by Paton et al. (2015) into weak and strong homogeneous granular targets representing planetary regoliths, that included the following materials, limestone powder, iron powder and sand. They found that sand had about half the strength of iron powder but was around ten times stronger than limestone powder. Here the layered targets include sili-beads on iron powder, iron powder on limestone powder, sand on iron powder and loose sand on compact sand.

Figure 17 shows the steps required to determine the impact speed and penetration distance using the shaft encoder for each target. First the shaft encoder signal is converted into distance summing its pulses and then into speed by dividing the distance corresponding to a pulse by the time. The accelerometer measurements in figure 18 shows some jittering due to vibration from the test rig chain rolling over the cogs. As a consequence variation a 
speed has been observed, when smoothing the derived speed from the shaft encoder, as having an amplitude of around $1 \mathrm{~cm} \mathrm{~s}^{-1}$. Oscillations are not apparent in the derived distance from the shaft encoder in figure 17 because the vertical displacement of the chain from the vibrations is small $(<1 \mathrm{~mm})$. The speed profiles feature a period of about one second where the test rig is accelerated up to impact speed followed by a brief coasting period lasting just under $0.1 \mathrm{~s}$ and then the impact which lasts between 0.1 to $0.2 \mathrm{~s}$. A model of the test rig's motion (equation 3) is shown fitted to the derived speed in figure 17. This allows for a more precise determination of the impact speed and position than the diagnostic measurements alone and demonstrates the test rig is working as required.

Figure 18 shows penetrometer and accelerometer measurements made during penetration of layered targets using X-PEN. The penetrometer measurements are shown superimposed on top of the accelerometer measurements to determine if there is any significant friction between the penetrometer shaft and the target. In figure 18 (a) the layered analogue regoliths consist of a $5 \mathrm{~cm}$ layer of sili-beads poured on top of a $15 \mathrm{~cm}$ layer of compacted iron powder. In figure 18 (b) the target consists of a $5 \mathrm{~cm}$ layer of iron powder sitting on a $5 \mathrm{~cm}$ layer of limestone powder which in turn is sitting on a 10 $\mathrm{cm}$ layer of iron powder. Schematics of the layered structure of the targets corresponding to figures 18 (a) and (b) can be found in figure 8. In figure 18 (c) the target consists of $5 \mathrm{~cm}$ of sand on top of $5 \mathrm{~cm}$ of iron powder on top of $10 \mathrm{~cm}$ of sand. In figure 18 (d) the target consists of $5 \mathrm{~cm}$ of loose sand, prepared by pouring, on top of $15 \mathrm{~cm}$ of compacted sand, prepared by tapping.

The impact into the first target, shown in figure 18 (a), results in a steprise increase to a plateau in the force that continues to a depth of $5 \mathrm{~cm}$ and is followed by a steep rise in force as the tip penetrates further. The penetrometer results in figure 18 (b) show an initial steep rise in the resistance to penetration as the tip impacts the top layer of iron powder followed by a region of a small rise in force as the tip passes through the limestone powder. The force then rises up steeply again as the tip passes through the lower iron powder layer. No discernable variation of force is detected during penetration from figure 18 (c). In figure 18 (d) there is negligible force while penetrating the loose sand layer followed by a steeper rise on entering the more compacted sand.

A low level plateau followed by a steep rise seen in figure 18 (a) can be explained as follows. The upper layer of sili-beads are a mono-disperse 

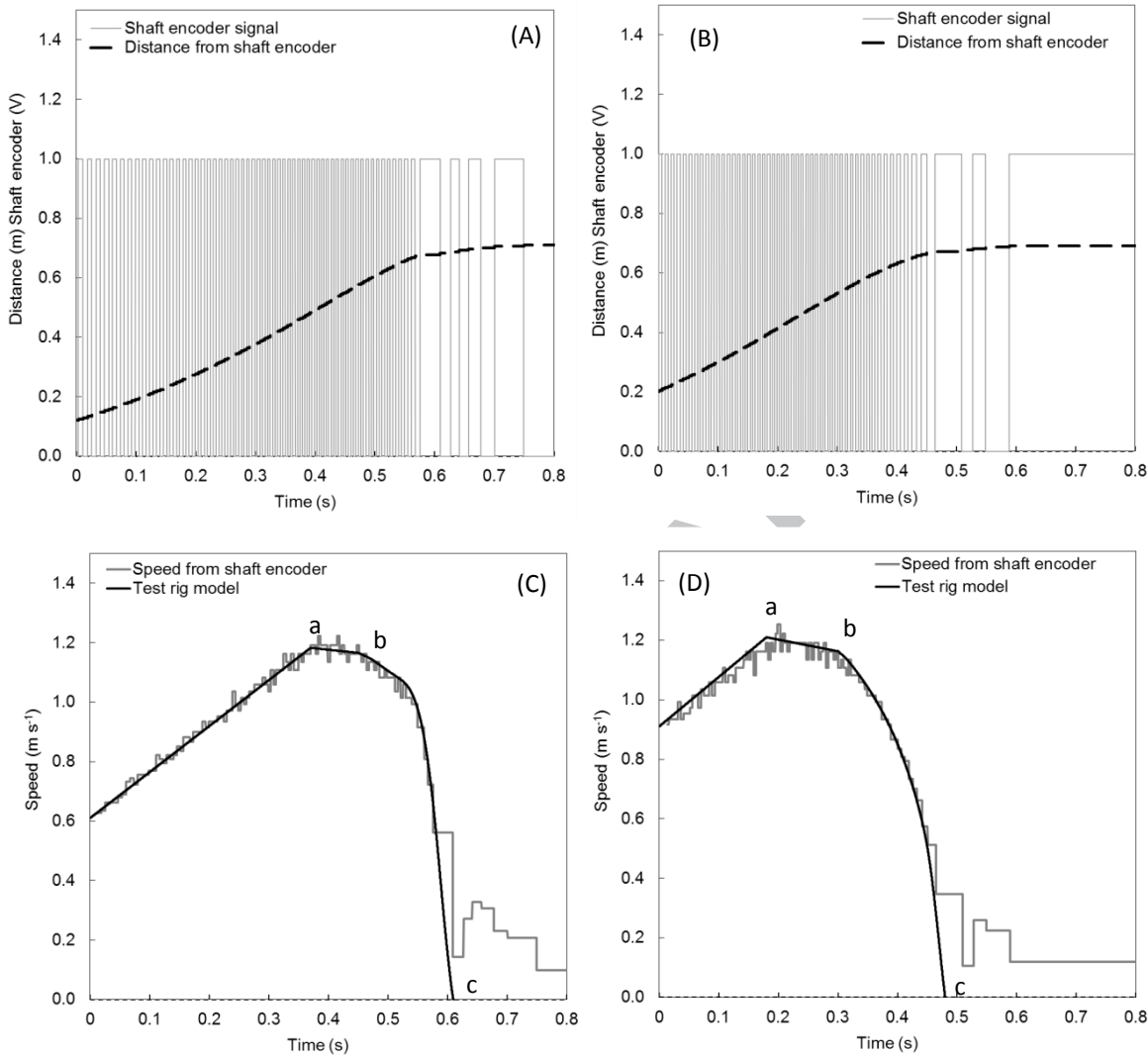

Figure 17: Shaft encoder derived information. The figure shows the shaft encoder signal together with the test rig distance and speed derived from the shaft encoder signal. The model of the test rig motion (equation 3 ) is compared to the test rig speed from the shaft encoder. (a) and (c) sili-bead - iron powder (b) and (d) iron powder - limestone powder iron powder. The impact speed was $1.2 \mathrm{~m} \mathrm{~s}^{-1}$ for (a) and (b). The penetration distance for all impacts was $16 \mathrm{~cm}$ except (a) which was $11 \mathrm{~cm}$. The points marked a, b and c on the charts represent the end of the acceleration phase, the end of the coasting phase and the end of the penetration respectively. 

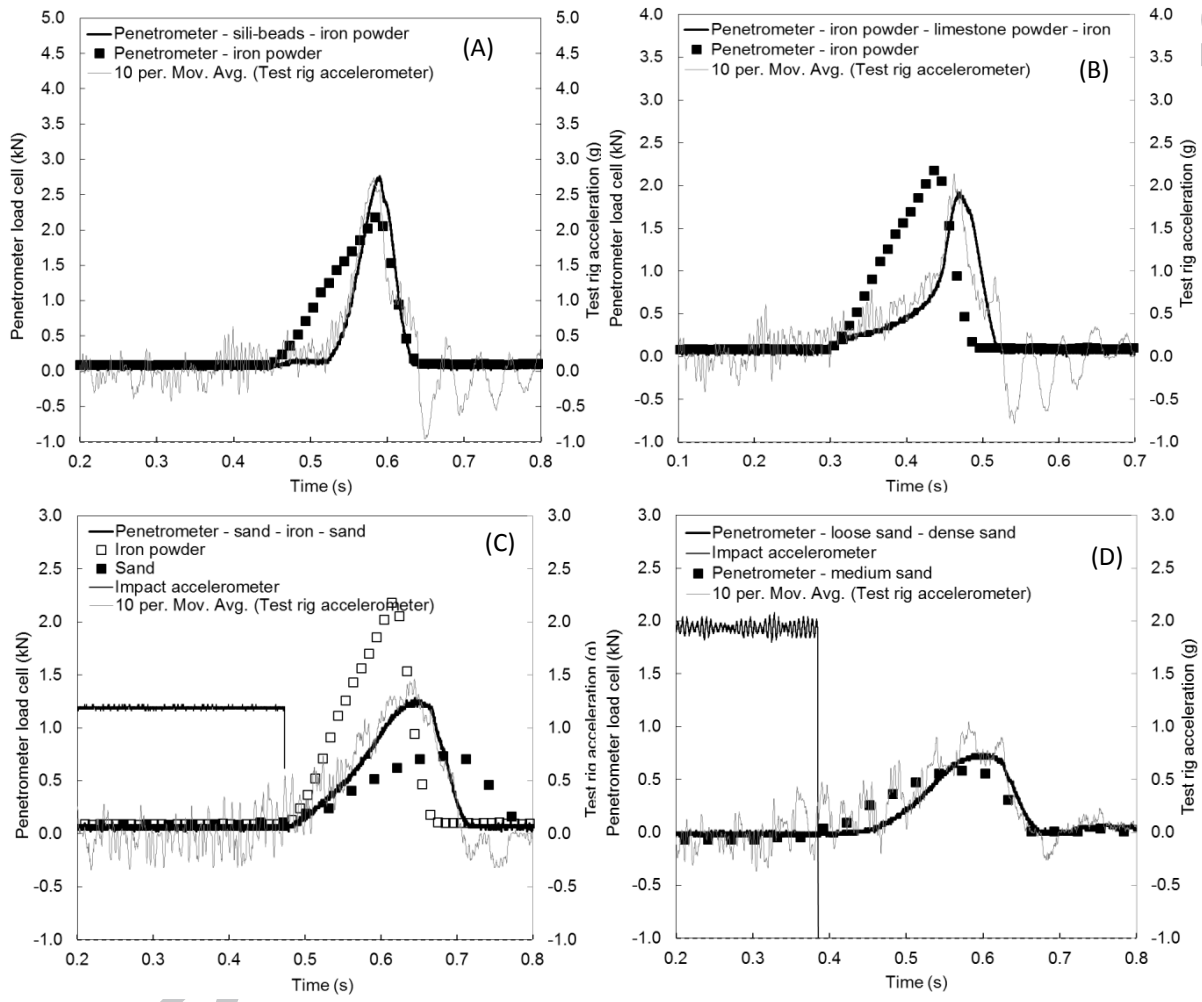

Figure 18: Comparison of accelerometer and penetrometer load cell measurements from the same test drop into layered targets. (a) sili-bead - iron powder (b) iron powder limestone powder - iron powder (c) sand - iron powder - sand (d) loose sand - compacted sand. The impact speed was $1.2 \mathrm{~m} \mathrm{~s}^{-1}$ for (a) and (b) and $0.86 \mathrm{~m} \mathrm{~s}^{-1}$ for (c) and (d). Results from impacts into homogenous compacted iron powder and sand are plotted for comparison. The vibration evident in the test rig accelerometer data is caused by the rig's chain running over the cogs. The surface contact (impact) accelerometer data, shown in (c) and (d), is used to determine when the penetrometer tip touches the surface. 
granular material consisting of $4 \mathrm{~mm}$ diameter spheres and are relatively weak whereas the lower layer consisting of iron powder is a strong material consisting of 10-100 micro-metre sized grains. Iron powder strength arises from the rough surface of its particles that causes significant intergranular friction. The steep-shallow-steep increase in force observed in figure 18 (b) can be explained as due to the iron powder, limestone and iron powder layering in the target.

The initial rise in the force in figure 18 (b) is steeper over the first $\mathrm{cm}$ of penetration into iron powder than the rest perhaps due to the accumulation of a mass of material in front of the tip. It is noted in figure 18 (b) that during penetration of the top layer of iron powder the increase in force is not as steep as when penetrating the bottom layer. This could be because limestone powder can be easily compacted as it consists of a wide range of particle sizes. It would then significantly weaken the response of the top layer of iron powder as it is easily pushed into the underlying layer of limestone. Alternatively, or in addition, the steep rise in force of the lower layer of iron powder could be influenced by the boundary of the container, particularly as the tip comes to rest within only a few centimetres of the container floor.

The relative lack of any significant deviation in the gradient in figure 18 (c) could be because the microstructural properties of sand and iron powder produce a similar response to penetration. The sand used in the target has a narrow range of grain sizes compared to iron powder which means it cannot be as easily compacted, but sand grains are highly spherical compared to iron powder grains suggesting they have less friction between the grains. Paton et al. (2015) found that sand had about half the strength of iron powder but was about 10 times stronger than limestone powder. The loose layer of sand in figure $18(\mathrm{~d})$ is not detected by the penetrometer and referral to the a surface contact accelerometer (section 3.1.3) is required to determine the start of penetration.

Forces on the penetrometer as measured by the accelerometer are similar to those as measured by the load cell, suggesting friction of the shaft has little effect, at least for shallow penetrations of up to $20 \mathrm{~cm}$ as tested here.

It may be difficult in practice to discern the properties of the top few centimetres of a regolith using a penetrometer for a number of reasons. A tip entry effect could occur where a compaction zone forms during tip entry causing the force to rise steeply with depth, which then levels off once the tip has penetrated. Alternatively, the mechanics of the regolith's top layer may be easily loosened during impact and could be misinterpreted as a loose 


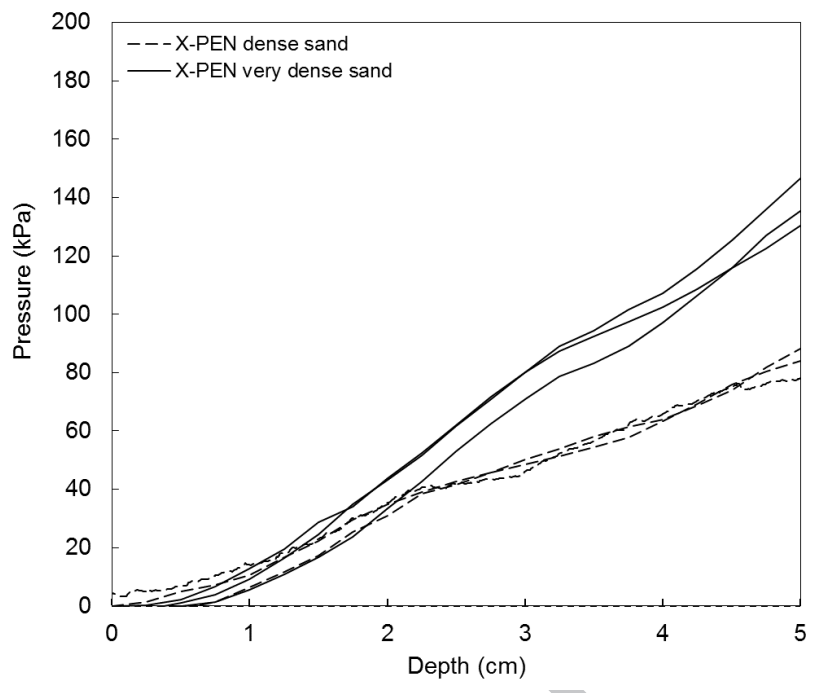

Figure 19: First few cm of penetration into sand.

layer when in fact it was in a more compacted state. Figures 19 and 20 show impacts into homogeneous iron powder and sand (Paton et al., 2015). Notice how in the first few $\mathrm{cm}$ of penetration in sand the rise in force is almost identical for dense and very dense sand targets. Similar results can be observed for iron powder in figure 20.

\subsection{Characterisation of granular materials}

To determine whether microstructural properties of the targets such as particle size and mass could be measured by X-PEN coarse-grained materials (gravel) of various sizes were impacted at a speed of $1 \mathrm{~m} \mathrm{~s}^{-1}$. These results were compared to measurements previously obtained in the same materials for ACC-E by Paton et al. (2012a). The impacts produced peaks in the data, e.g. figures 23 and 26, the number and magnitude of which are identified and measured in the same way as described in Paton et al. (2012a). The X-PEN measurements were also designed to determine a trend of forcedepth gradients with target particle diameters to help investigate the odd negative gradient in the Huygens penetrometry data. The data from X-PEN is compared to a model based on the one developed by Paton et al. (2012a) (section 4.2).

Figure 21 demonstrates the ability of the penetrometer (X-PEN) to determine particle diameter. The number of peaks in the X-PEN data increases 


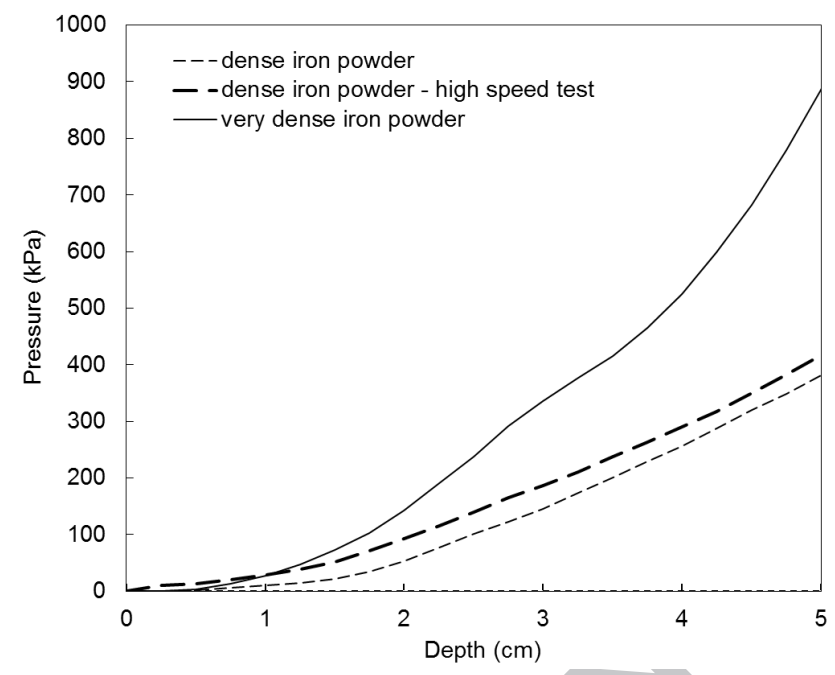

Figure 20: First few $\mathrm{cm}$ of penetration into iron powder.

with increasing average particle diameter. This is the opposite trend to that found by Paton et al. (2012a) for the ACC-E penetrometer which is also shown in figure 21. The opposite trend is because the impact forces from the smallest particles are not being sensed due to the more massive, less responsive tip and lower impact speed of X-PEN compared to ACC-E. XPEN impacted at a speed of $1 \mathrm{~m} \mathrm{~s}^{-1}$ compared to tests with ACC-E which impacted the target at $5 \mathrm{~m} \mathrm{~s}^{-1}$. This would reduce the impact force by a factor of about five. Figure 22 demonstrates the ability of X-PEN to sense the particle mass. The summed peak magnitude increases with increasing average particle mass which is a similar result in Paton et al. (2012a). The magnitude is lower than for ACC-E because or X-PEN's lower sensitivity to impacts.

Figure 23 shows the force-depth profiles from penetration of gravel targets by X-PEN. The notable characteristics of the data are increasing peak magnitudes with increasing average particle size and the appearance of larger bumps in the background force, i.e. a less smoothly increasing force with depth. It can also be seen in figure 23 that for targets with larger particles the force-depth gradient is less steep than for targets with smaller particles.

Figure 24 plots the pressure-depth gradient against the particle-tip diameter ratio for X-PEN and compares the results to the hybrid DEM. Pressure rather than force is used here to enable the comparison of results from $\mathrm{X}$ - 


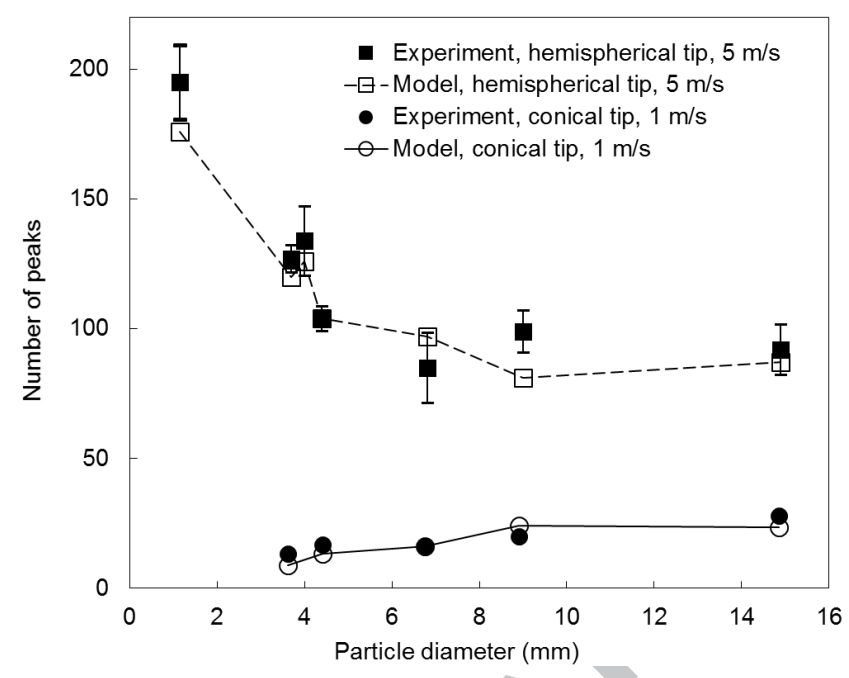

Figure 21: Number of peaks identified in the impact force signature from penetrations of X-PEN into gravel of different particles sizes at $1 \mathrm{~m} \mathrm{~s}^{-1}$ compared to those found by Paton et al. (2012a) for ACC-E into the same targets.

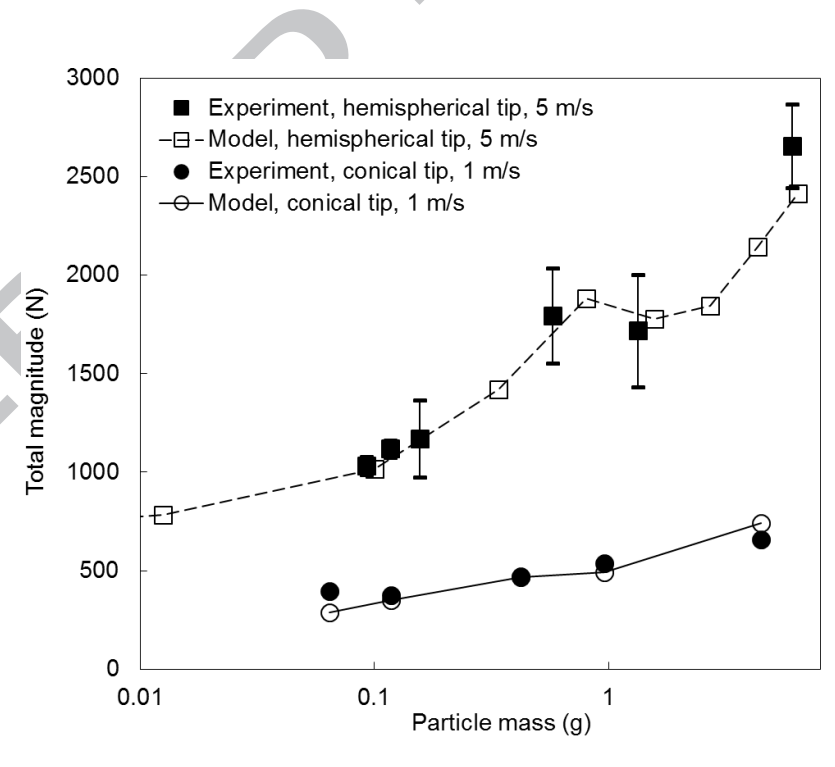

Figure 22: Sum of the magnitudes of the peaks identified in the impact force signature from penetrations by X-PEN into gravel of different particles masses at $1 \mathrm{~m} \mathrm{~s}^{-1}$ compared to those calculated for peaks in data from the hemispherical tipped penetrometer ACC-E. 

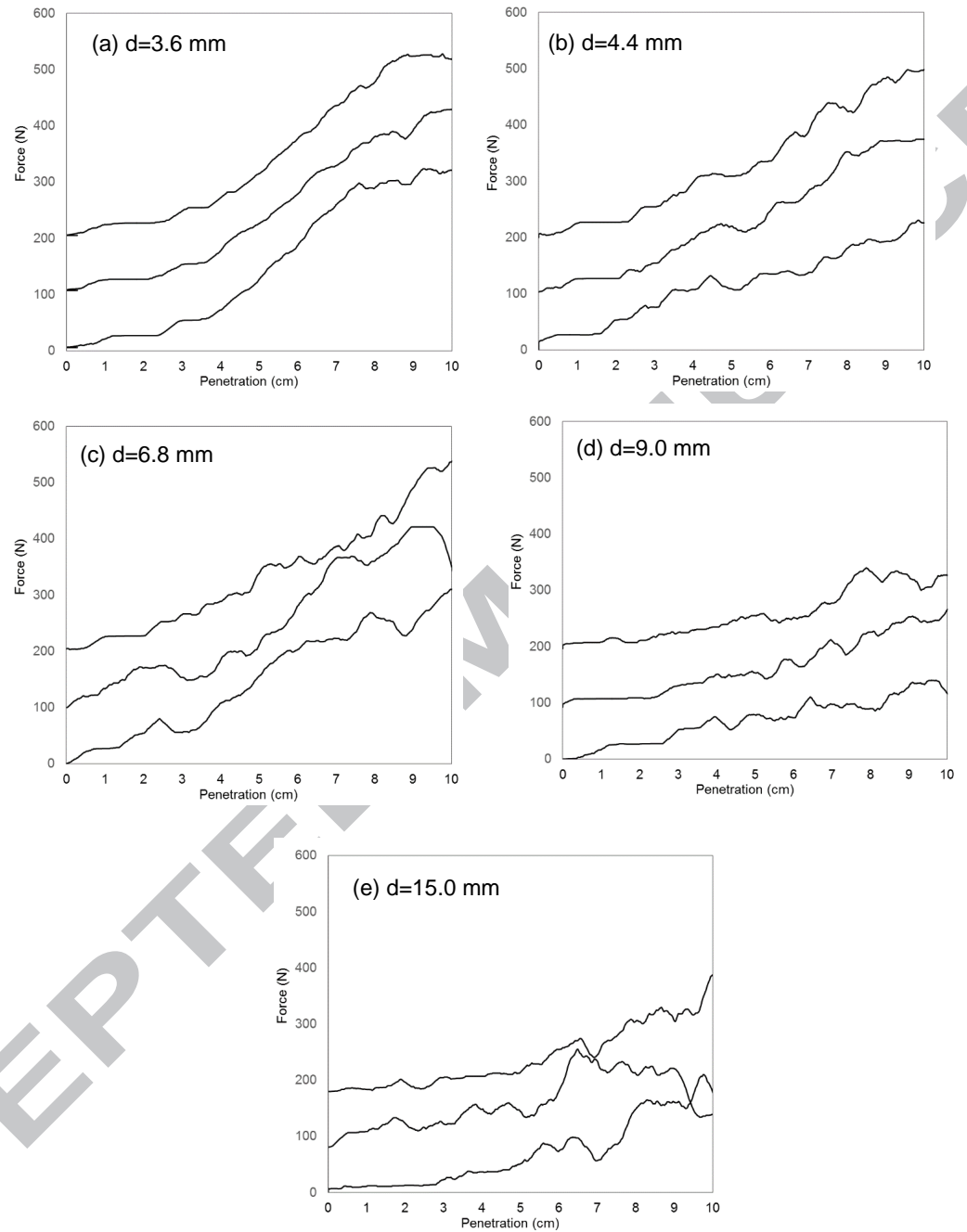

Figure 23: Impact forces measured for X-PEN penetrations of five regolith analogues with different mean particle sizes at a speed of $1 \mathrm{~m} \mathrm{~s}^{-1}$. Three impact signatures, offset by 100 $\mathrm{N}$, are shown for each analogue. 


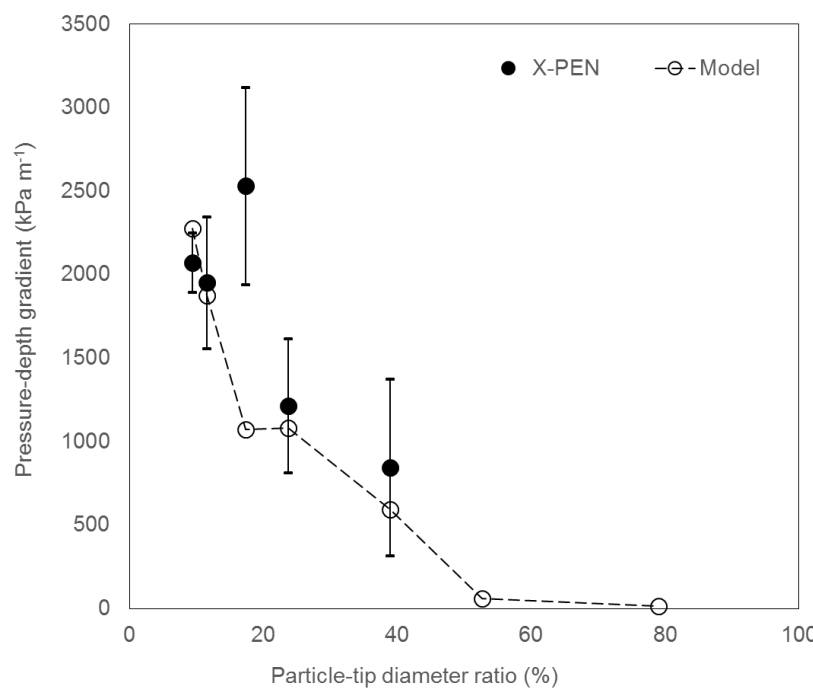

Figure 24: Gradient of pressure with depth for X-PEN measurements in gravel compared to the model. The $\mathrm{x}$-axis is the ratio of particle diameter to tip diameter. The average diameter of the particles in the targets are 3.6, 4.4, 6.8, 9.0 and $14.9 \mathrm{~mm}$. The diameter of the penetrometer is $38 \mathrm{~mm}$. Five impacts into each target were made.

PEN with ACC-E and refers to the force normal to the sensor divided by the area of the tip's base. The particle-tip diameter ratio was used rather than simply the particle diameter to help interpret the results in terms of the penetrometer design, i.e. is there a tip size effect? In figure 24 it can be seen the pressure-depth gradient decreases with increasing particle-tip diameter ratio as suggested in figure 23. The hybrid DEM matches the results from X-PEN in most cases. Extrapolating the model to particle-tip diameter ratios greater than $50 \%$ with the model predicts a close to zero force-depth gradient for targets with particles approaching a particle-tip diameter ratio of $100 \%$.

A notable outlier in figure 24 is the force-depth gradient for the target with an average particle diameter of $6.8 \mathrm{~mm}$. The particle shape for this target is more prolate, i.e. cylindrical, while the other targets have more disc shaped particles. This difference may explain its strong resistance to penetration.

A decreasing pressure-depth gradient with increasing particle-tip diameter ratio is interpreted as the target responding as a weak cellular-type material for targets when the voids are comparable in size to the penetrometer tip. In other words, for large particles the penetrometer can advance by rearranging 
a few neighbouring particles rather than setting into motion a whole mass of material around the tip that can form a shearing plane, as with smaller particles, e.g. Puech \& Foray (2002).

Figure 25 shows the pressure-depth gradient plotted against the particletip diameter ratio for impacts of ACC-E into the same granular targets as used for X-PEN in figure 24. The standard deviation in figure 25 for ACC$\mathrm{E}$ is much larger (by about a factor of four) than for X-PEN in figure 24. A possible explanation is as follows. A hemispherical tip is blunter than a conical tip making it more difficult for the particles to flow over the tip. A blunt tip may be more sensitive to the packing arrangement of the particles at the surface when the particle diameters are a large fraction of the tip diameter, i.e. if a blunt tip impacts a large particle almost head on the resulting force at the start of penetration will belarge compared to a conical tip whose point would likely pass to the side of the particle.

At first glance the overall trend in figure 25 appears to indicate an increasing pressure-depth gradient with particle-tip diameter ratio. On closer inspection it is noted that the second point from the origin, representing the average pressure-depth gradient for $4.4 \mathrm{~mm}$ gravel, from the origin is below the first point, representing $3.6 \mathrm{~mm}$ gravel. These two points roughly coincide with the downward trend observed in figure 24 . The three points of averages furthest from the origin in figure 25 are unusually high compared to the results in figure 24.

A possible explanation for the large pressure-depth gradients in figure 25 could be that on occasion ACC-E encounters high forces when deeper in the target from particles that are in a jammed state arising from localised force chains (Cates et al., 1999; Stone et al., 2004). The penetrometer then experiences a large force as it overcomes the force chain. For targets with smaller particles this may not be apparent because of the formation of a compaction zone and the mobilsation of particles in front of the tip. Large force-depth gradients were not observed with the conical tipped X-PEN possibly for this reason, as the tip is bigger than ACC-E. Also it may be the case that a blunt tip tends to slam down directly onto a force chain if the particles are large compared to the tip.

An important aspect of figure 25 in regards to interpreting the Huygens data is that three out of five of the impacts into the targets with a particle diameter of $4.4 \mathrm{~mm}$ produced a negative force-depth gradient. The gradient for two of the tests are particularly close to the ACC-E measurement from Titan. One of these have been plotted in figure 26 (b) for comparison with 


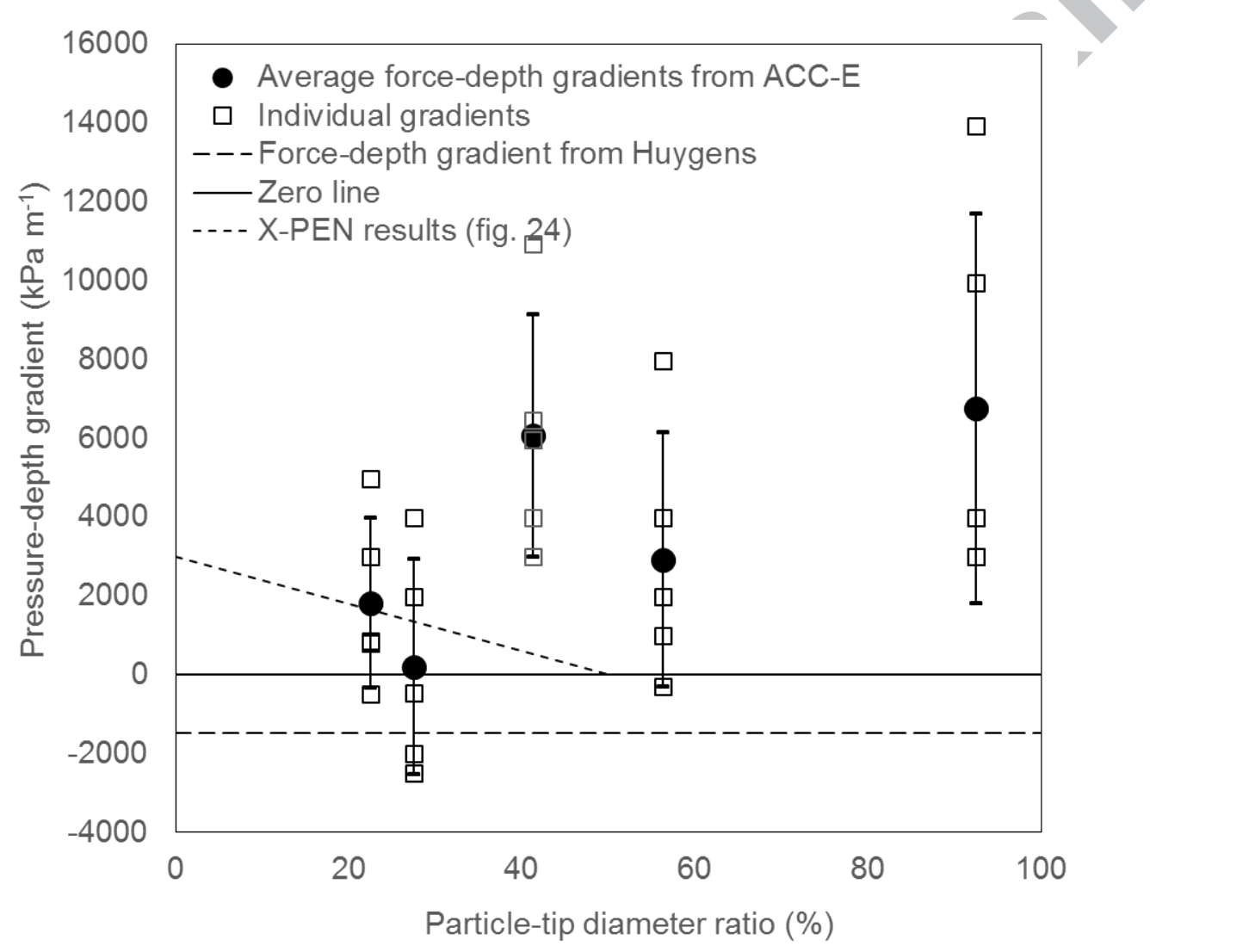

Figure 25: Gradient of pressure with depth for ACC-E measurements in gravel. The dotted line represents the negative force-depth gradient from the Huygens penetrometer measurement on Titan. The $\mathrm{x}$-axis is the ratio of particle diameter to tip diameter. The average diameter of the particles in the targets are 3.6, 4.4, 6.8, 9.0 and $14.9 \mathrm{~mm}$. The diameter of the penetrometer is $16 \mathrm{~mm}$. Five measurements were made by ACC-E in each target. 
the Huygens penetrometry measurement. Other particle diameters, i.e. 3.6 and $9.0 \mathrm{~mm}$, can also produce negative force-depth gradients however these appear to have a lower magnitude and occur less frequently than for $4.4 \mathrm{~mm}$ diameter gravel. The force-depth profile obtain by ACC-E for 3.6 and 9.0 $\mathrm{mm}$ gravel are shown in figure $26(\mathrm{c})$ and $(\mathrm{d})$.

An attempt to reproduce the negative force-depth gradient as seen in the Huygens' data was made with our hybrid DEM. A similar profile to the force-depth profile could be reproduced by having a decrease in the number of particle-particle impacts being felt by the tip from 10 over the upper half of the depth to 5 over the lower half of penetration. This may suggest that there is an increased mobilisation of surface material at impact which is inhibited with depth due to the confining forces until the inertial forces are dampened after which the resistance to penetration is mostly due to static forces, i.e. friction between the particles. The large peak at the start of penetration in the Huygens ACC-E signal suggests the mobilisation of a large mass perhaps an ice pebble with a diameter of $3 \mathrm{~cm}$.

\section{Conclusions}

We have investigated aspects of penetrometer design for use on an asteroid in terms of tip size and shape. We analysed the Huygens penetrometer data from Titan as a motivation for the study. Our focus was on the unusual decreasing resistance with depth observed in the Titan subsurface by the ACC-E penetrometer, which is relatively small hemispherical tip $16 \mathrm{~mm}$ in diameter. We deduced that this downward trend may not be so unusual and could be a result of the interaction of the penetrometer, i.e. a tip entry effect, with a gravel target consisting of particles with a diameter of around $4 \mathrm{~mm}$.

An experimental impact penetrometer (X-PEN), which has a large conical tip $38 \mathrm{~mm}$ in diameter, was tested in layered targets and the results were used to determine if layering could be used to interpret the Huygens' penetrometer data. X-PEN and ACC-E were tested in gravel targets and the results used to understand aspects of penetrometer design such as tip size and shape. The measurements also proved useful for interpreting the Huygens' data.

$\mathrm{X}-\mathrm{PEN}$ readily detected layering in targets that consists of materials whose strengths are significantly different from each other. All tests resulted in positive force-depth profiles and so were not considered representative of the Huygens penetrometer signal. 

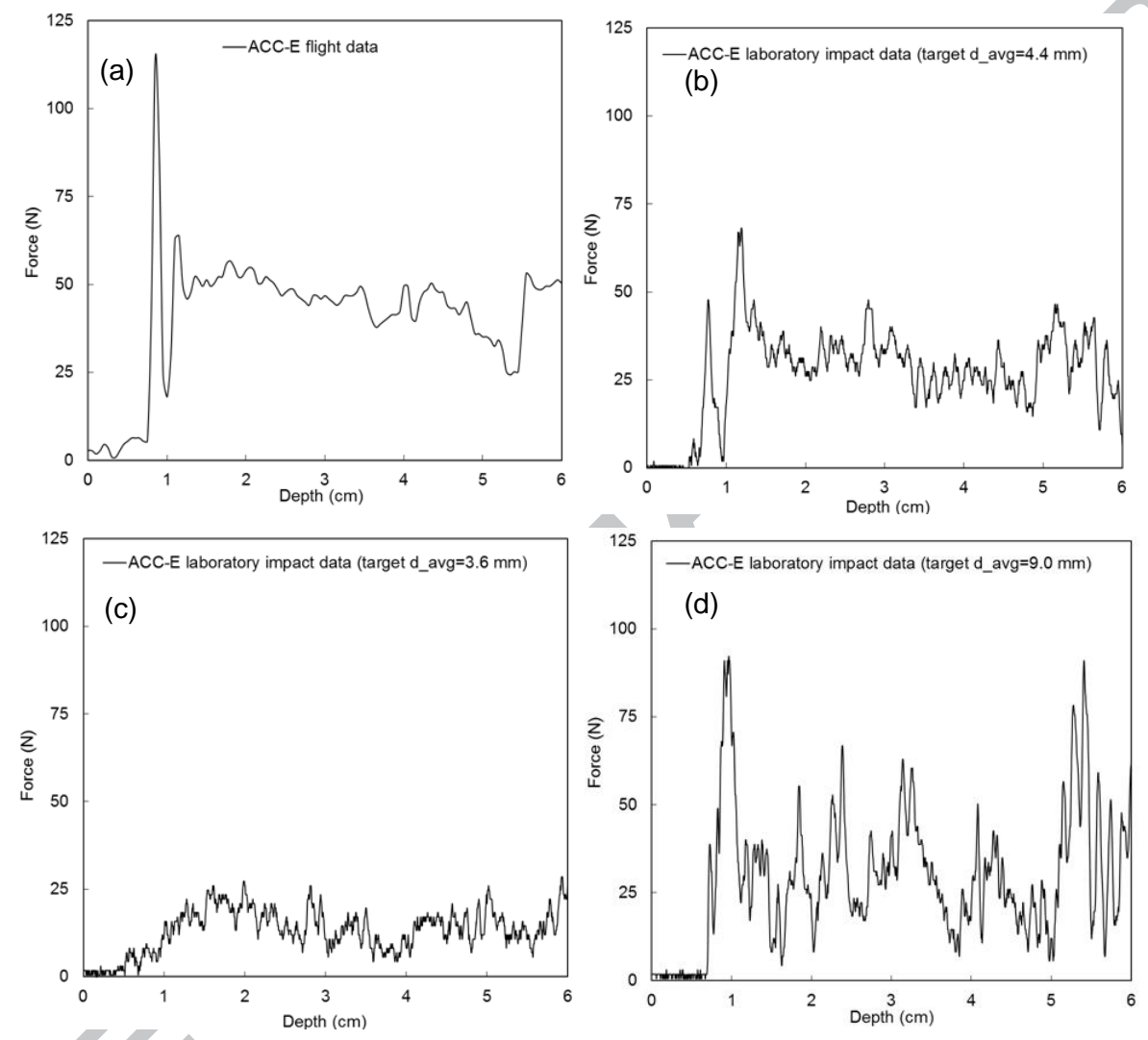

Figure 26: Huygens SSP ACC-E penetrometry data (a) compared with laboratory data in gravel with an average diameters of $4.4 \mathrm{~mm}$ (b), $3.6 \mathrm{~mm}$ (c) and $9.0 \mathrm{~mm}$ (d). The negative gradients for 3.6 and $9.0 \mathrm{~mm}$ shown in figure 25 were calculated using data shown in (c) and (d). The gradient in the laboratory data was calculated from a depth of 1.5 to $5.5 \mathrm{~cm}$ which corresponds to the region of decreasing force in the ACC-E flight data. 


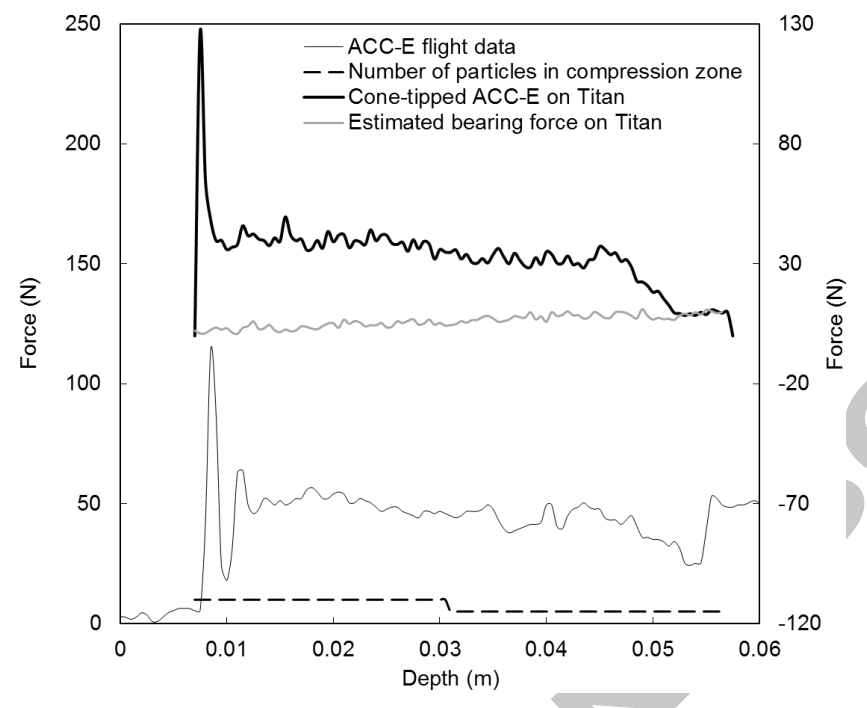

Figure 27: Huygens SSP ACC-E penetrometry data compared with a DEM model of ACC-E impacting $4.4 \mathrm{~mm}$ diameter ice gravel with a solid density of $920 \mathrm{~kg} \mathrm{~m}^{-3}$. The simulated target contained 3240 particles. The large peak at the begining is generated from an impact with a $3 \mathrm{~cm}$ pebble. The bearing strength model due to friction is shown under the impact model.

A decrease of the force-depth gradient with increasing average particle size was observed with X-PEN impacts in gravel targets. The results with ACC-E and X-PEN suggest that the resistance to penetration is sensitive to the ratio of particle to tip diameter with the resistance decreasing with increasing particle size. The fact that X-PEN did not produce a downward slope of resistance with depth in any of the tests, but ACC-E did in several targets, suggests it is an effect also related to tip shape.

The downward trend in the ACC-E data was interpreted using a hybrid DEM as the mobilisation of a large number of particles when the tip enters the target. The number of particles mobilised then decreases, possibly due to the confining pressure of the Titan regolith, as ACC-E penetrates deeper.

We also investigate the rebound potential of a landing spacecraft in a low gravity environment when fitted with a penetrometer. A simulated spacecraft with a large penetrating device with similar dimensions to X-PEN attached to its base, appears to dampen the elastic response of the spacecraft so preventing a significant rebound as with a spacecraft fitted without a penetrometer.

We recommend that the diameter of an impact penetrometer with a hemi- 
spherical tip for measuring particle microstructural properties be at least twice the diameter of the average particle diameter expected in the target to enable a more straightforward interpretation of the data. A conical tip appears to be less sensitive to tip entry effects in gravel. This is likely due to its more slender shape that allows it to gently pass large particles. A large penetrometer could possibly be ustilised for managing the rebound of a spacecraft impacting regolith on a body with substantial gravity.

\section{Acknowledgements}

The authors wish to acknowledge the Hosie Bequest to the Royal Astronomical Society that funded this work. We thank Ralph Lorenz for his helpful comments.

\section{References}

Atkinson, K. R., Zarnecki, J. C., Towner, M. C., Ringrose, T. J., Hagermann, A., Ball, A. J., Leese, M. R., Kargl, G., Paton, M. D., Lorenz, R. D., \& Green, S. F. (2010). Penetrometry of granular and moist planetary surface materials: Application to the Huygens landing site on Titan. Icarus, 210, 843-851.

Ball, A., Garry, J., Lorenz, R., \& Kerzhanovich, V. (2010). Planetary Landers and Entry Probes.

Burr, D. M., Emery, J. P., Lorenz, R. D., Collins, G. C., \& Carling, P. A. (2006). Sediment transport by liquid surficial flow: Application to Titan. Icarus, $181,235-242$.

Cates, M. E., Wittmer, J. P., Bouchaud, J., \& Claudin, P. C. (1999). Jamming and static stress transmission in granular material. Chaos, 3, 511522 .

Cherkasov, I. I., \& Shvarev, V. V. (). Soviet investigations of the mechanics of lunar soils. Soil Mechanics and Foundation Engineering, 10, 252-256.

Chitu, C. C., Stefanescu, R. M., Muresan, T., Barcinski, T., Seweryn, K., Getka, M., Ortega, C., Galipienzo, J., \& Visentin, G. (2015). Design and development of an active landing gear system for low-gravity environments. Oct 14, 2015 - IAC-15 66th International Astronautical Congress. 
Colwell, J. E., Sture, S., Cintala, M., Durda, D., Hendrix, A., Goudie, T., Curtis, D., Ashcom, D. J., Kanter, M., Keohane, T., Lemos, A., Lupton, M., \& Route, M. (2008). Ejecta from impacts at $0.22 .3 \mathrm{~m} / \mathrm{s}$ in low gravity. Icarus, 195, 908-917.

Goldman, D. I., \& Umbanhowar, P. (2008). Scaling and dynamics of sphere and disk impact into granular material. Physical Review E, 7r, 1-2.

Hilchenbach, M. (2004). Simulation of the landing of rosetta philae on comet 67p/churyumov-gerasimenko. SIMPACK USER MEETING 2004, Nov 910, Wartburg, Eisenach, Germany.

Hilchenbach, M., Rosenbauer, H., \& Chares, B. (2004). First contact with a comet surface: Rosetta Lander simulations. In L. Colangeli, E. Mazzotta Epifani, \& P. Palumbo (Eds.), The New Rosetta Targets. Observations, Simulations and Instrument Performances (p. 289). volume 311 of Astrophysics and Space Science Library.

Jones, R. H. (1971). Lunar surface mechanical properties from Surveyor data. Journal of Geophysical Research, 76, 7833-7843.

Kargl, G., Kömle, N. I., Ball, A. J., \& Lorenz, R. D. (2009). In Penetrometry in the Solar System II, Graz, September 25-28, 2006, Austrian Academy of Science Press.

Keller, H. U., Grieger, B., Küppers, M., Schröder, S. E., Skorov, Y. V., \& Tomasko, M. G. (2008). The properties of Titan's surface at the Huygens landing site from DISR observations. Planetary and Space Science, 56, $728-752$.

Kömle, N. I., Kargl, G., Ball, A. J., \& Lorenz, R. D. (2001). In Penetrometry in the Solar System, Graz, October 18-20, 1999, Austrian Academy of Science Press.

Lorenz, R., Bannister, M., Daniell, P., Krysinski, Z., Leese, M., Miller, R., Newton, G., Rabbetts, P., Willett, D., \& Zarnecki, J. (1994). An impact penetrometer for a landing spacecraft. Measurement and Science Technology, 5, 1033-1041.

Lorenz, R., Hibbard, K., Paul, M. V., Walsh, J., Olds, D. W., Willits, B. E. B., S., \& Kretsch, W. E. (2015). Instrumented splashdown testing 
of a scale model Titan Mare Explorer (TiME) capsule. The Aeronautical Journal, 119, 409-431.

Moore, H. J., \& Jakosky, B. M. (1989). Viking landing sites, remote-sensing observations, and physical properties of Martian surface materials. Icarus, $81,164-184$.

Paillet, F. L., \& Saunders, W. R. (1990). Geophysical Applications for Geotechnical Investigations.

Paton, M. D., Green, S. F., \& Ball, A. J. (2008). Measuring the properties of asteroid regolith subsurface particles using a penetrometer.. In Asteroid, Comets and Meteors, Baltimore, 13-18 July 2008.

Paton, M. D., Green, S. F., \& Ball, A. J. (2012a). Microstructural penetrometry of asteroid regolith analogues and Titan's surface. Icarus, 220, 787-807.

Paton, M. D., Green, S. F., Ball, A. J., Zarnecki, J. C., \& Harri, A. M. (2015). Using the inertia of spacecraft during landing to penetrate regoliths of the Solar System. Advances in Space Research, 56, 1242 - 1263.

Paton, M. D., Harri, A. M., Mkinen, T., \& Green, S. F. (2012b). Investigating thermal properties of gas-filled planetary regoliths using a thermal probe. Geosci. Instrum. Method. Data Syst., 1, 7-21.

Poletto, F., Magnani, P., Gelmi, R., Corubolo, P., Re, E., Schleifer, A., Perrone, A., Salonico, A., \& Coste, P. (2015). Seismic While Drilling (SWD) methodology in support to Moon subsurface stratigraphy investigations. Acta Astronautica, 110, 99-114.

Popov, V. L. (2010). Contact mechanics and friction: physical principles and applications.

Puech, A., \& Foray, P. (2002). Refined model for interpreting shallow penetration cpts in sands. In Proceedings of the Annual Offshore Technology Conference. Society of Petroleum Engineers.

Putzig, N. E., Mellon, M. T., Kretke, K. A., \& Arvidson, R. E. (2005). Global thermal inertia and surface properties of Mars from the MGS mapping mission. Icarus, 173, 325-341. 
Reid, R., Roveda, L., Nesnas, B., \& Pavone, M. (2014). Contact dynamics of internally-actuated platforms for the exploration of small solar system bodies. Presented at i-SAIRAS, 2014, Quebec, Canada.

Schröder, S. E., Karkoschka, E., \& Lorenz, R. D. (2012). Bouncing on Titan: Motion of the Huygens probe in the seconds after landing. Planetary and Space Science, 73, 327-340.

Seiff, A., Stoker, C. R., Young, R. E., Mihalov, J. D., McKay, C. P., \& Lorenz, R. D. (2005). Determination of physical properties of a planetary surface by measuring the deceleration of a probe upon impact: Application to Titan. Planetary and Space Science, 53, 594-600.

Seweryn, K., Skocki, K., Banaszkiewicz, M., Grygorczuk, J., Kolano, M., Kuciski, T., Mazurek, J., Morawski, M., Biaek, A., Rickman, H., \& Wawrzaszek, R. (2014). Determining the geotechnical properties of planetary regolith using low velocity penetrometers. Planetary and Space Science, 99, $70-83$.

Spohn, T., Knollenberg, J., Ball, A. J., Banaszkiewicz, M., Benkhoff, J., Grott, M., Grygorczuk, J., Hüttig, C., Hagermann, A., Kargl, G., Kaufmann, E., Kömle, N., Kührt, E., Kossacki, K. J., Marczewski, W., Pelivan, I., Schrödter, R., \& Seiferlin, K. (2015). Thermal and mechanical properties of the near-surface layers of comet $67 \mathrm{P} /$ Churyumov-Gerasimenko. Science, 349 .

Spohn, T., Seiferlin, K., Hagermann, A., Knollenberg, J., Ball, A. J., Banaszkiewicz, M., Benkhoff, J., Gadomski, S., Gregorczyk, W., Grygorczuk, J., Hlond, M., Kargl, G., Kührt, E., Kömle, N., Krasowski, J., Marczewski, W., \& Zarnecki, J. C. (2007). Mupus: A Thermal and Mechanical Properties Probe for the Rosetta Lander Philae. Space Science Reviews, 128, 339-362.

Stone, M. B., Barry, R., Bernstein, D. P., Pelc, M. D., Tsui, Y. K., \& Schiffer, P. (2004). Local jamming via penetration of a granular medium. Phys. Rev. E, 70, 041301.

Sullivan, R., Arvidson, R., Bell, J. F., Gellert, R., Golombek, M., Greeley, R., Herkenhoff, K., Johnson, J., Thompson, S., Whelley, P., \& Wray, J. (2008). Wind-driven particle mobility on Mars: Insights from Mars Exploration 
Rover observations at "El Dorado" and surroundings at Gusev Crater. Journal of Geophysical Research (Planets), 113, 6.

Thiel, M., Stöcker, J., Rohe, C., Kömle, N. I., Kargl, G., Hillenmaier, O., \& Lell, P. (2003). The ROSETTA Lander anchoring system. In R. A. Harris (Ed.), 10th European Space Mechanisms and Tribology Symposium (pp. 239-246). volume 524 of ESA Special Publication.

Yano, H., Kubota, T., Miyamoto, H., Okada, T., Scheeres, D., Takagi, Y., Yoshida, K., Abe, M., Abe, S., Barnouin-Jha, O., Fujiwara, A., Hasegawa, S., Hashimoto, T., Ishiguro, M., Kato, M., Kawaguchi, J., Mukai, T., Saito, J., Sasaki, S., \& Yoshikawa, M. (2006). Touchdown of the Hayabusa Spacecraft at the Muses Sea on Itokawa. Science, 312, 1350-1353.

Zarnecki, J. C., Banaszkiewicz, M., Bannister, M., Boynton, W. V., Challenor, P., Clark, B., Daniell, P. M., Delderfield, J., English, M. A., Fulchingnoni, M., Garry, J. R. C., Geake, J. E., Green, S. F., Hathi, B., Jaroslawski, S., Leese, M. R., Lorenz, R. D., McDonnell, J. A. M., Merryweather-Clarke, N., Mill, C. S., Miller, R. J., Newton, G., Parker, D. J., Rabbetts, P., Svedhem, H., Turner, R. F., \& Wright, M. J. (1997). The Huygens Surface Science Package. In A. Wilson (Ed.), Huygens: Science, Payload and Mission (p. 177). volume 1177 of ESA Special Publication.

Zarnecki, J. C., Leese, M. R., Garry, J. R. C., Ghafoor, N., \& Hathi, B. (2002). Huygens' Surface Science Package. Space Science Reviews, 104, 593-611.

Zarnecki, J. C., Leese, M. R., Hathi, B., Ball, A. J., Hagermann, A., Towner, M. C., Lorenz, R. D., McDonnell, J. A. M., Green, S. F., Patel, M. R., Ringrose, T. J., Rosenberg, P. D., Atkinson, K. R., Paton, M. D., Banaszkiewicz, M., Clark, B. C., Ferri, F., Fulchignoni, M., Ghafoor, N. A. L., Kargl, G., Svedhem, H., Delderfield, J., Grande, M., Parker, D. J., Challenor, P. G., \& Geake, J. E. (2005). A soft solid surface on Titan as revealed by the Huygens Surface Science Package. Nature, 438, 792-795.

\section{Appendix A. Numerical penetrometer-particle mechanical model}

When the tip and the particle collide they are deformed in a direction normal to the local surfaces in contact. 


$$
F_{\text {max }}=1.28 \frac{v_{z}^{1.2}}{x^{0.4}}\left(\frac{m_{1} m_{2}}{m_{1}+m_{2}}\right)^{0.6}\left(r_{1} r_{2}\right)^{0.1}
$$

where $m_{1}$ and $m_{2}$ are the masses of the colliding bodies, $r_{1}$ and $r_{2}$ are local radii of curvature on the tip at the impact point and the radius of the particle respectively and $X$ is defined as in the following equation.

$$
X=\frac{1-\sigma_{1}^{2}}{E_{1}}+\frac{1-\sigma_{2}^{2}}{E_{1}}
$$

where $\sigma_{1}$ and $\sigma_{2}$ are the Poisson ratios and $E_{1}$ and $E_{2}$ are the elasticity of the tip and the particle. The duration of contact between a sphere and a cylinder, from Hertz's theory of impact, is as follows.

$$
\Delta t_{p k}=2.86 \frac{X^{0.4}}{v_{z}^{0.2}}\left(\frac{m_{1} m_{2}}{m_{1}+m_{2}}\right)^{0.6}\left(r_{1} r_{2}\right)^{-0.1}
$$

The geometry of the impact with the conical tip introduces a sin $\alpha$ factor into the equation to account for the compression force transferred vertically through the pentrometer's sensor.

$$
F_{\text {max }}=1.28 \frac{\left(v_{z} \sin \alpha\right)^{1.2}}{x^{0.4}}\left(\frac{m_{1} m_{2}}{m_{1}+m_{2}}\right)^{0.6}\left(r_{1} r_{2}\right)^{0.1}(\sin \alpha+\mu \cos \alpha)
$$

Damped harmonic motion is used to model the motion of the tip. The tip-sensor is considered as a spring-mass system.

$$
m \frac{d^{2} x}{d t^{2}}+\beta \frac{d x}{d t}+\omega_{0}^{2} x=F_{d}
$$

where $\beta$ is the damping coefficient, $\mathrm{d}$ is the displacement from the equilibrium position, $\omega_{0}$ is the angular frequency and $t$ is the time. The angular frequency is defined as follows.

$$
\omega_{0}=\left(\frac{k_{e f f}}{m}\right)^{0.5}
$$

where $m$ is the mass of the oscillating tip and $k_{e f f}$ is the effective spring constant. The spring constant is calculated from the elasticity of the sensor which is defined as follows. 


$$
k_{e f f}=\frac{E A}{l}
$$

where $E$ is the elasticity, $l$ is the thickness of the sensor and $A$ is the area of the sensor in contact with the penetrometer.

$$
\frac{d^{2} x}{d t^{2}}=\frac{1}{m_{t i p}}\left(F_{d}-\beta v_{t}+x_{t} k_{e f f}\right)
$$

where $m_{t i p}$ is the mass of the tip, $F_{d}$ is the driving force from the impacting particle, $v_{t}$ is the velocity of the tip relative to the rest of the penetrometer at time $t$ and $x_{t}$ is the displacement of the tip relative to its equilibrium position. The force on the tip is as follows.

$$
\begin{gathered}
F_{d}=F_{\max } \sin (\omega t) \\
t<t_{p k}
\end{gathered}
$$

where $F_{\max }$ is the maximum magnitude of a peak generated by an impacting particle as defined in equation A.4 and the angular frequency is defined as follows,

$$
\omega=\frac{\pi}{t_{p k}}
$$

The acceleration of the tip is calculated using an iterative method as follows.

$$
a_{t}=\frac{1}{m_{t i p}}\left(F_{t-\delta t}-\beta v_{t-\delta t}-x_{t-\delta t} k_{e f f}\right)
$$

where

$$
\begin{aligned}
& v_{t}=v_{t-\delta t}+a_{t-\delta t} \delta t \\
& z_{t}=z_{t-\delta t}+v_{t-\delta t} \delta t
\end{aligned}
$$

At the beginning of each time step the force acting on the tip is calculated as follows,

$$
F_{i}=F_{\max } \sin \left(\pi \frac{t_{i-1}-t_{c}}{\Delta t_{p k}}\right)
$$




$$
t_{i}-t_{c}<\Delta t_{p k}
$$

where $t_{c}$ is the time at which the particle first makes contact with the penetrometer tip. When the simulation is initialised each particle is assigned a location and the penetrometer is assigned a location and a velocity. The start of impact begins when the distance between the centre of the particle and the centre of the tip base is equal to the tip radius plus the radius of the hemisphere. At this point $t_{c}=0$ and equation A.15 can be used to calculate the force. If there are two or more impacts at the same time then the force on the tip is calculated as follows,

$$
\begin{gathered}
\sum_{j=1}^{j=n_{t i p}} F_{j} \sin \left(\pi \frac{t_{i-1}-t_{j}}{\Delta t_{j}}\right) \\
t_{i}-t_{c}<\Delta t_{j}
\end{gathered}
$$

where $n_{\text {tip }}$ is the number of particles in contact with the tip. When the particles sliding over the surface of the tip are comparable in size to the tip the forces transferred to these particles from shearing forces will become intermittent. There will be occasions when a large part of the tip enters a void and there will be nothing to push against causing a drop in the force. We can represent this intermittent force on the tip by quantising the shearing force, i.e. dividing the force defined in equation A.15, as follows.

$$
F_{p}=\frac{N \rho g z}{n_{p}}
$$

where $n_{p}$ is the average number of particles in contact with the tip, i.e. $n_{p}=A_{t i p} / A_{p}$ where $A_{t i p}$ is the surface area of the tip and $A_{p}$ is the crosssection area of a particle. Equation A.17 can then be modified as follows,

$$
\sum_{j=1}^{j=n_{t i p}} F_{j} \sin \left(\pi \frac{t_{i-1}-t_{j}}{\Delta t_{j}}\right)+n_{t i p} F_{p}
$$

